\title{
Electronic dynamic Hubbard model: exact diagonalization study
}

\author{
J. E. Hirsch \\ Department of Physics, University of California, San Diego \\ La Jolla, CA 92093-0319
}

(Dated: October 24, 2018)

\begin{abstract}
A model to describe electronic correlations in energy bands is considered. The model is a generalization of the conventional Hubbard model that allows for the fact that the wavefunction for two electrons occupying the same Wannier orbital is different from the product of single electron wavefunctions. We diagonalize the Hamiltonian exactly on a four-site cluster and study its properties as a function of band filling. The quasiparticle weight is found to decrease and the quasiparticle effective mass to increase as the electronic band filling increases, and spectral weight in one- and two-particle spectral functions is transfered from low to high frequencies as the band filling increases. Quasiparticles at the Fermi energy are found to be more 'dressed' when the Fermi level is in the upper half of the band (hole carriers) than when it is in the lower half of the band (electron carriers). The effective interaction between carriers is found to be strongly dependent on band filling becoming less repulsive as the band filling increases, and attractive near the top of the band in certain parameter ranges. The effective interaction is most attractive when the single hole carriers are most heavily dressed, and in the parameter regime where the effective interaction is attractive, hole carriers are found to 'undress', hence become more like electrons, when they pair. It is proposed that these are generic properties of electronic energy bands in solids that reflect a fundamental electron-hole asymmetry of condensed matter. The relation of these results to the understanding of superconductivity in solids is discussed.
\end{abstract}

PACS numbers:

\section{INTRODUCTION}

The fact that the Coulomb repulsion between two electrons in a doubly occupied atomic orbital is smaller than the value predicted from the expectation value of the Coulomb interaction with single electron wavefunctions establishes that atomic orbitals are not infinitely rigid[1]: electrons will develop intra-atomic correlations to reduce their Coulomb repulsion. This well-known fact is not incorporated in the conventional single band Hubbard model[2], which assumes that two electrons of opposite spin will doubly-occupy the same single electron atomic orbital. Dynamic Hubbard models[3] attempt to remedy this deficiency by either introducing an auxiliary boson degree of freedom to mimic the orbital relaxation that occurs on double atomic occupancy, or by allowing more than one atomic orbital. Here we consider a purely electronic dynamic Hubbard model with two orbitals per site and no auxiliary boson degree of freedom.

The model considered here has some superficial resemblance to various multi-orbital Hubbard models that have been considered in the past such as the degenerate Hubbard model[沟, the Falicov-Kimball model[ [] and the Anderson lattice model [6]. However conceptually it is rather different. The goal is not to model the physics of electrons in degenerate atomic orbitals, nor of two different partially occupied bands in a solid, nor of local magnetic moments interacting with conduction electrons, nor of mixed valence. Rather, we are interested in modeling the physics of a single band, and based on general arguments [3] argue that it is necessary, to understand single band physics, to include at least one other higherlying orbital besides the one that is being filled as the electronic band is being filled. This second orbital becomes increasingly important as the filling of the band increases beyond one half.

When an electronic energy band is less than half-full, electrons can avoid each other by developing elaborate interatomic correlations. For this situation the ordinary single band Hubbard model may be adequate. However, when the band is more than half-filled, some Wannier orbitals are necessarily doubly occupied. The resulting large cost in Coulomb energy cannot be avoided by any interatomic correlations; instead, it can be reduced by the electrons developing intra-atomic correlations. The conventional single band Hubbard model cannot describe this, hence it is inadequate to describe electronic energy bands that are more than half-full: it is necessary to introduce another degree of freedom. Here this is achieved by having a second atomic orbital in the model, that becomes increasingly occupied as the band filling increases beyond half filling. As we will see in this paper, the resulting electron-hole asymmetry in the band causes the quasiparticle weight of carriers in the upper half of the band (holes) to be smaller than that in the lower half of the band (electrons), and their effective mass to be larger. We believe that this physics is generally a part of the physics of electronic energy bands in solids. Its quantitative importance will depend on the particular solid under consideration.

In section II we introduce the electronic dynamic Hubbard model to be studied in this paper. Section III gives analytic results in limiting cases, and section IV presents 
numerical results for effective interaction, quasiparticle weight and effective mass as a function of band filling and model parameters. Section V present results for optical absorption, and in Sect. VI we discuss the relation between the parameters in the Hamiltonian and atomic parameters in materials. We conclude in Sect. VII with a discussion of the applicability of these results to the description of real electrons in real solids.

\section{MODEL HAMILTONIAN}

The static (conventional) single band Hubbard model is defined by the Hamiltonian

$$
H=-\sum_{i, j, \sigma} t_{i j} c_{i \sigma}^{\dagger} c_{j \sigma}+U \sum_{i} n_{i \uparrow} n_{i \downarrow}
$$

which properly emphasizes the fact that the intra-atomic electron-electron repulsion is the dominant source of electronic correlation in solids. Here, $c_{i \sigma}^{\dagger}$ creates an electron in a Wannier orbital centered at lattice site $i$, and the hopping amplitude $t_{i j}$ is the Fourier transform of the single electron band energy $\epsilon_{k}$ :

$$
t_{i j}=\frac{1}{N} \sum_{k} e^{i k\left(R_{i}-R_{j}\right)} \epsilon_{k} .
$$

The fundamental problem with the Hubbard Hamiltonian Eq. (1) is that it implicitly assumes that the state of two electrons in a Wannier orbital is a single Slater determinant $c_{i \uparrow}^{\dagger} c_{i \downarrow}^{\dagger} \mid 0>$, with $\mid 0>$ the state of the empty orbital. This is incorrect, precisely because of the existence of the electron-electron interaction described by the Hubbard $U$. We extend the Hamiltonian Eq. (1) to allow for the fact that when two electrons occupy the same Wannier orbital, intra-orbital electronic correlations will develop due to the large local electron-electron repulsion. This effect can be described by having at least two Wannier orbitals per site, which the electrons will partially occupy to reduce their Coulomb repulsion. Let $c_{i \sigma}^{\dagger}$ and $c_{i \sigma}^{\dagger}$ be the operators creating electrons into these Wannier orbitals. We consider the local Hamiltonian [7]

$$
\begin{aligned}
H_{i}= & U n_{i \uparrow} n_{i \downarrow}+U^{\prime} n_{i \uparrow}^{\prime} n_{i \downarrow}^{\prime}+\epsilon n_{i}^{\prime}+V n_{i} n_{i}^{\prime} \\
& -t^{\prime} \sum_{\sigma}\left(c_{i \sigma}^{\dagger} c_{i \sigma}^{\prime}+h . c .\right)
\end{aligned}
$$

with $n_{i \sigma}=c_{i \sigma}^{\dagger} c_{i \sigma}, n_{i \sigma}^{\prime}=c_{i \sigma}^{\dagger} c_{i \sigma}^{\prime}, \quad n_{i}=n_{i \uparrow}+n_{i \downarrow}$, $n_{i}^{\prime}=n_{i \uparrow}^{\prime}+n_{i \downarrow}^{\prime}$. In the extreme tight binding limit the Wannier orbitals become atomic orbitals, and we will cast the discussion in that language even though it should be more generally applicable.

The primed orbital is higher in energy by $\epsilon$ than the unprimed orbital, hence more extended in space, so that the Coulomb repulsion $U^{\prime}$ should be smaller than $U$. The orbital energy $\epsilon$ will generally satisfy the relation $t<\epsilon<U$, so that when the site is doubly occupied electrons will partially occupy this orbital to reduce their Coulomb repulsion and there is no justification for neglecting this second orbital as is done in the Hamiltonian Eq. (1). $V$ describes the Coulomb repulsion between one electron in each orbital, and $t^{\prime}$ the intra-atomic hybridization between these orbitals. The lattice Hamiltonian is then

$$
\begin{aligned}
H= & -\sum_{i j}\left[t_{i j} c_{i \sigma}^{\dagger} c_{j \sigma}+t_{i j}^{\prime}\left(c_{i \sigma}^{\dagger} c_{j \sigma}^{\prime}+h . c .\right)+t_{i j}^{\prime \prime} c_{i \sigma}^{\dagger} c_{j}^{\prime}(3] \mathrm{b}\right) \\
& +\sum_{i} H_{i}
\end{aligned}
$$

with $H_{i}$ given by Eq. (3a). We expect the various hopping matrix elements $t_{i}, t_{i j}^{\prime}, t_{i j}^{\prime \prime}$ to be similar in magnitude and hence will assume for the remainder of this paper

$$
t_{i j}=t_{i j}^{\prime}=t_{i j}^{\prime \prime}
$$

and furthermore that the hopping connects only nearest neighbor sites, hence $t_{i j} \equiv t$ for $i, j$ nearest neighbors, zero otherwise.

It should be pointed out that we do not expect the qualitative physics of the model to depend on the particular choice for the hoppings Eq. (4). For example, in Ref. [7] the same site Hamiltonian Eq. (3a) was considered with hoppings $t_{i j}^{\prime}=t_{i j}^{\prime \prime}=0$ instead of Eq. (4), and the same qualitative physics was obtained as in the model considered here. Certainly, quantitative differences will occur depending on these choices that should be studied in the future. In this connection it is also relevant to note that in a recent study of the periodic Anderson model, different choices of the hybridization (whether same site or neighboring sites) were found to not affect the underlying physics 8 .

\section{ANALYTIC RESULTS}

\section{A. Non-interacting band structure}

The single electron part of the Hamiltonian Eq. (3) is, after Fourier transforming

$$
\begin{gathered}
H=\sum_{k}\left(\begin{array}{cc}
c_{k \sigma}^{\dagger} & c_{k \sigma}^{\prime \dagger}
\end{array}\right) H_{k}\left(\begin{array}{c}
c_{k \sigma} \\
c_{k \sigma}^{\prime}
\end{array}\right) \\
H_{k}=\left(\begin{array}{cc}
\epsilon_{k} & \epsilon_{k}-t^{\prime} \\
\epsilon_{k}-t^{\prime} & \epsilon_{k}+\epsilon
\end{array}\right)
\end{gathered}
$$

with

$$
\epsilon_{k}=-2 t \cos k
$$

for a one-dimensional geometry. Two bands result, with energy versus $k$ relation

$$
E_{k}^{1,2}=\epsilon_{k}+\frac{\epsilon}{2} \pm \sqrt{\left(\frac{\epsilon}{2}\right)^{2}+\left(\epsilon_{k}-t^{\prime}\right)^{2}}
$$


The two bands are separated by an indirect gap

$$
\begin{aligned}
\Delta= & E_{k=0}^{2}-E_{k=\pi}^{1}=\sqrt{\left(\frac{\epsilon}{2}\right)^{2}+\left(2 t-t^{\prime}\right)^{2}}+ \\
& \sqrt{\left(\frac{\epsilon}{2}\right)^{2}+\left(2 t+t^{\prime}\right)^{2}}-4 t
\end{aligned}
$$

which is always positive for $\epsilon>0$. The bandwidth of the two bands is approximately given by $D_{1}=4 t+2 t^{\prime}$, $D_{2}=4 t-2 t^{\prime}$. Figure 1 shows the band structure for various parameters. Note that the effective mass in the lower band is larger near the top of the band than near the bottom; this effect becomes much more pronounced in the presence of electron-electron interactions.

The zero temperature single electron spectral function for the system with $N$ electrons is

$$
\begin{aligned}
& A_{\alpha}(k, \omega)=\sum_{n}\left|<n_{N+1}\right| c_{\alpha k \sigma}^{\dagger}\left|0_{N}>\right|^{2} \times \\
& \delta\left(\omega-\left(E_{n}^{N+1}-E_{0}^{N+1}+\mu_{N}\right)\right)+ \\
&\left|<n_{N-1}\right| c_{\alpha k \sigma}\left|0_{N}>\right|^{2} \delta\left(\omega+\left(E_{n}^{N-1}-E_{0}^{N-1}-\mu_{N-1}\right)\right)
\end{aligned}
$$

Here, $\left|n_{N}>\right|$ is the n-th excited state of the system with $N$ electrons ( $n=0$ is the ground state) and $E_{n}^{N}$ is the eigenvalue, and $\mu_{N}=E_{0}^{N+1}-E_{0}^{N}, \mu_{N-1}=E_{0}^{N}-$ $E_{0}^{N-1}$. For a metal $\mu_{N}=\mu_{N-1} \equiv \mu$ and we redefine the frequency $\omega \rightarrow \omega+\mu$ so that

$$
\begin{aligned}
A_{\alpha}(k, \omega)= & \sum_{n}\left|<n_{N+1}\right| c_{\alpha k \sigma}^{\dagger}\left|0_{N}>\right|^{2} \times \\
& \delta\left(\omega-\left(E_{n}^{N+1}-E_{0}^{N+1}\right)\right)+ \\
\mid & <n_{N-1}\left|c_{\alpha k \sigma}\right| 0_{N}>\left.\right|^{2} \delta\left(\omega+\left(E_{n}^{N-1}-E_{0}^{N-1}\right)\right)
\end{aligned}
$$

The index $\alpha$ labels the fermion operator of the unprimed or primed orbital, or an appropriate linear combination theerof. The creation operator for an electron in band 1 is given by

$$
c_{1 k \sigma}=u_{k} c_{k \sigma}+v_{k} c_{k \sigma}^{\prime}
$$

with $u_{k}, v_{k}$ obtained from diagonalization of $H_{k}$, Eq. (5b). Using this operator in Eq. (10) we have simply

$$
A_{1}(k, \omega)=\delta\left(\omega-\left(E_{k}^{1}-\mu\right)\right)
$$

and the quasiparticle weight for a single electron in this band is the coefficient of the $\delta$-function in Eq. (12)

$$
z_{k}=1
$$

\section{B. Quasiparticle weight for interacting system}

In an interacting many-body system the spectral function has the form

$$
A_{\alpha}(k, \omega)=z_{k} \delta\left(w-\left(\epsilon_{k}-\mu\right)\right)+A_{\alpha}^{\prime}(k, \omega)
$$

where $0 \leq z_{k} \leq 1$ is the quasiparticle weight, and $A_{\alpha}^{\prime}$ is the incoherent part of the spectral function. We define the quasiparticle weight in our model at the Fermi energy by

$$
z(N)=\left|<O_{N-1}\right| c_{\alpha k_{F} \sigma}\left|O_{N}>\right|^{2}
$$

with the quasiparticle operator defined by

$$
c_{\alpha k_{F} \sigma}=u_{k_{F}} c_{k_{F} \sigma}+v_{k_{F}} c_{k_{F} \sigma}^{\prime}
$$

with $u_{k}^{2}+v_{k}^{2}=1$. We choose the particular linear combination that maximizes the quasiparticle weight Eq. (15). The following simple relations are easily proven:

$$
z(N)=\sum_{i}\left[\left|<O_{N-1}\right| c_{i \sigma}\left|O_{N}>\right|^{2}+\left|<O_{N-1}\right| c_{i \sigma}^{\prime}\left|O_{N}>\right|^{2}\right]
$$

$$
u_{k_{F}}^{2}=\frac{\sum_{i}\left|<O_{N-1}\right| c_{i \sigma}\left|O_{N}>\right|^{2}}{z(N)}
$$

$$
v_{k_{F}}^{2}=\frac{\sum_{i}\left|<O_{N-1}\right| c_{i \sigma}^{\prime}\left|O_{N}>\right|^{2}}{z(N)}
$$

Eq. (17) gives the total quasiparticle weight at the Fermi energy, and $1-z$ gives the amount of spectral weight in the incoherent part of the spectral function. The quantities $u_{k_{F}}, v_{k_{F}}$ indicate how much of the quasiparticle resides in the lower and upper orbital respectively. For a single band conventional Hubbard model the quasiparticle weight at the Fermi energy is given by the first term in Eq. (17).

\section{Optical conductivity and effective mass}

The current operator in our model is given by

$$
J=i t \sum_{i}\left[\left(c_{i+1 \sigma}^{\dagger}+c_{i+1 \sigma}^{\dagger}\right)\left(c_{i \sigma}+c_{i \sigma}^{\prime}\right)-h . c .\right]
$$

and we will compute the optical conductivity at zero temperature given by

$$
\sigma_{1}(\omega)=\pi \sum_{m} \frac{|<0| J|m>|^{2}}{E_{m}-E_{0}} \delta\left(\omega-\left(E_{m}-E_{0}\right)\right)
$$

The optical sum rule states that the integral of the optical conductivity is $[9]$

$$
\int_{0}^{\infty} d \omega \sigma_{1}(\omega)=\frac{\pi}{2}<0|-T| 0>
$$

with the kinetic energy given by

$$
T=-t \sum_{i \sigma \delta}\left(c_{i \sigma}^{\dagger}+c_{i \sigma}^{\dagger}\right)\left(c_{i+\delta \sigma}+c_{i+\delta \sigma}^{\prime}\right)
$$


In our two-orbital model, optical transitions include both 'intraband' transitions as well as interband transitions to the second band. For parameters where a clear separation of energy scales occurs one can define an effective Hamiltonian describing the low energy part of the Hilbert space, and write a 'partial' conductivity sum rule

$$
\left.\int_{0}^{\omega_{m}} d \omega \sigma_{1}(\omega)=A_{l}=\frac{\pi}{2}<0\left|-T_{e f f}\right| 0\right\rangle
$$

where $T_{\text {eff }}$ is the kinetic energy in the lower band, and the high frequency cutoff $\omega_{m}$ excludes transitions to the second band.

\section{Strong coupling limit}

We consider the Hamiltonian Eq. (3) in the parameter regime

$$
\begin{aligned}
& U^{\prime}+2 \epsilon<V+\epsilon<U \\
& U, U^{\prime}, V>>\epsilon>>t^{\prime}
\end{aligned}
$$

These conditions ensure that a single electron at a site resides primarily in the lower orbital, while in the doubly occupied site two electrons of opposite spin reside primarily in the higher orbital. Some results in this limit were discussed in ref. [7]. The site eigenstates to lowest order in $t^{\prime}$ are

$$
\begin{gathered}
|\tilde{\uparrow}>=| \uparrow>|0>+\delta| 0>\mid \uparrow> \\
|\tilde{\downarrow}>=| 0>\mid \uparrow \downarrow>+\delta^{\prime}[|\uparrow>| 0>+|0>\downarrow>| 0>]
\end{gathered}
$$

where the first (second) ket in the product refers to the lower (higher) orbital, and

$$
\begin{gathered}
\delta=t^{\prime} / \epsilon \\
\delta^{\prime}=\frac{t^{\prime}}{V-U^{\prime}-\epsilon}
\end{gathered}
$$

The single site quasiparticle weight for a hole is, from Eq. (17)

$$
z_{h}=|<\tilde{\downarrow}| c_{\uparrow}|\tilde{\uparrow} \downarrow>|^{2}+|<\tilde{\downarrow}| c_{\uparrow}^{\prime}|\tilde{\uparrow} \downarrow>|^{2}=\left(\delta+\delta^{\prime}\right)^{2} \equiv S^{2}
$$

and the single site quasiparticle weight for an electron is

$$
z_{e}=|<0| c_{\uparrow}|\tilde{\uparrow}>|^{2}+|<0| c_{\uparrow}^{\prime}|\tilde{\uparrow}>|^{2}=1
$$

Hence the single site spectral function for a hole has other terms in addition to the quasiparticle term since $z_{h}<$ 1 , while the site spectral function for an electron is a single $\delta$-function. Consequently, in the solid the spectral function for a single electron in the ground state of the Hamiltonian Eq. (3) is a $\delta$-function, while the spectral function for a single hole in the lower band has incoherent contributions, as discussed in ref. [3].

We can define quasiparticle operators for the lower band $\tilde{c}_{i \sigma}$ as operators connecting the site ground states

$$
\begin{aligned}
& \tilde{c}_{i \sigma}^{\dagger}|0>=| \tilde{\sigma}> \\
& \tilde{c}_{i \sigma}^{\prime \dagger}|0>=| \tilde{\uparrow}>
\end{aligned}
$$

and the relation between bare electron and quasiparticle operators is 10

$$
c_{i \sigma}=\left[1+(S-1) \tilde{n}_{i,-\sigma}\right] \tilde{c}_{i \sigma}
$$

Replacing the bare fermion operators in the kinetic energy Eq. (3b) in terms of the quasiparticle operators yields an effective Hamiltonian for quasiparticles in the lower band, of the form

$$
\begin{aligned}
H_{e f f}= & -t \sum_{i j, \sigma}\left[1+(S-1)\left(\tilde{n}_{i,-\sigma}+\tilde{n}_{j,-\sigma}\right)+\right. \\
& \left.(S-1)^{2} \tilde{n}_{i,-\sigma} \tilde{n}_{j,-\sigma}\right]\left(\tilde{c}_{i \sigma}^{\dagger} \tilde{c}_{j \sigma}+\text { h.c. }\right)
\end{aligned}
$$

This Hamiltonian gives rise to a 'correlated hopping' term which leads to pairing of holes. The enhancement of the hopping amplitude for a hole when the sites involved in the hopping process have an additional hole is

$$
\Delta t=t S(1-S)
$$

and leads to superconductivity as discussed in detail elsewhere 11.

\section{NUMERICAL RESULTS}

We diagonalize the Hamiltonian Eq. (3) on an $N=4$ site lattice with number of electrons $N_{e}$ ranging from 0 to 8 , as appropriate to fill the lower band. The maximum number of states in the Hilbert space is 4900. To reduce the computational complexity and because we will be interested in the case of large Coulomb interactions we discard all states where there are more than two electrons at any given site from the Hilbert space. We also discard states that have two electrons of the same spin at a site, because we will not be interested in spin-polarized states. This simplification should not change the qualitative physics of interest here, and such states certainly become irrelevant in the limit of large interorbital repulsion $V$; however it could have a quantitative effect for small $V$ and should be investigated further. With these simplifications the maximum size of the Hilbert space is 1024 states when there are 6 electrons in the four-site system. 
Figure 2 shows schematically the band under consideration and the number of states in the Hilbert space for different positions of the Fermi level. Note that there are many more states in the Hilbert space for a given number of holes in the band than for the same number of electrons. This fact alone indicates that the system will be more incoherent when the band is more than half filled compared to when it is less than half filled. The figure shows one representative state in each sector: when there is one electron at the site, it resides primarily in the lower orbital, when there are two electrons at the site they are depicted as occupying the higher orbital which will be the dominant contribution in the parameter range of interest.

\section{A. Choice of boundary conditions}

For the small system under study boundary conditions are of course important. We have calculated properties of the system using periodic, antiperiodic and free ends boundary conditions (bc). While periodic bc may yield results closer to the thermodynamic limit in some cases, we believe free ends bc are preferable for several reasons. Most importantly, the single particle eigenstates for the finite chain are non-degenerate with free ends bc, while degenerate states occur for both periodic and antiperiodic bc.

Consider the effective interaction between two electrons in the chain with $N_{e}$ electrons, defined as

$$
U_{e f f}\left(N_{e}\right)=E_{0}\left(N_{e}\right)+E_{0}\left(N_{e}-2\right)-2 E_{0}\left(N_{e}-1\right)
$$

with $E_{0}\left(N_{e}\right)$ the ground state energy with $N_{e}$ electrons. Figure 3 shows the effective interaction versus band occupation for various parameter values. For all the different boundary conditions the effective interaction becomes attractive near the top of the band for certain parameter values. This is a robust effect. On the other hand, it can be seen that the effective interaction can be negative for the half-filled band case for periodic bc, and it is zero near the bottom of the band for antiperiodic bc. These are spurious effects related to finite system degeneracies, that are expected to dissappear in the thermodynamic limit [12]. Instead, for free ends bc the effective interaction is repulsive except near the top of the band. This behavior should persist for larger systems. Quantitatively, we expect the magnitude of the attractive interaction to be somewhat smaller for the infinite system than for the small cluster 13.

Another reason to use free ends boundary conditions is that the optical sum rule is satisfied in that case for the finite system [14], while if periodic boundary conditions are used the 'Drude weight' needs to be added by hand to the optical response. Furthermore, the expression for the quasiparticle weight Eq. (17) is not correct when there are degenerate states at the Fermi energy. Hence we will use free ends boundary conditions for the remainder of this paper.

\section{B. Quasiparticle bands}

We begin our study with a look at the energy level spectrum of the model. We choose sets of parameters that yield a clear separation of the spectrum of the lower band states. Figure 4 shows the energy levels as a function of $N_{e}$, the number of electrons in the cluster, for a non-interacting case (a) and for an interacting case (b). The dashed line separates the low-lying band 'intraband' states from the other states. For the noninteracting case, the spectrum in the lower band is nearly symmetric for electrons and holes, as one would expect. Instead, with electron-electron interactions a large electron-hole asymmetry exists.

Consider first the states for a single electron $\left(N_{e}=1\right)$ and for a single hole $\left(N_{e}=7\right)$. There are 4 states in each case ( $=$ the number of sites in the cluster) and the distance between the lowest and highest intraband state is the bandwidth. Clearly, for the interacting case the hole bandwidth is much smaller than the electron bandwidth. The effective mass of the quasiparticle is inversely proportional to the spacing between intraband states. Clearly, the quasihole is substantially heavier than the quasielectron in the presence of electron-electron interactions for these parameter values.

As the number of electrons or of holes is increased, the number of intraband states increases. There are 6 intraband states for the half-filled band $\left(N_{e}=4\right)$, and 12 states for all other occupations, because states with double site occupancy are pushed to much higher energies due to the large Coulomb repulsions. Note the asymmetry in the spectra in the interacting case for band filling less and more than one half: the quasiparticle band is substantially narrower when the carriers are holes (more than half-filled band) compared to the corresponding case when the carriers are electrons. In the effective strong coupling Hamiltonian one obtains a quasiparticle bandwidth that decreases monotonically as the electronic band filling increases:

$$
D\left(n_{e}\right)=D\left[1-\frac{n_{e}}{2}(1-S)\right]^{2}
$$

with $0 \leq n_{e}=N_{e} / N \leq 2$ the band filling.

\section{Quasiparticle properties versus band filling}

We consider first a case where the second band is well separated in energy, with $\epsilon=10$. Consider the evolution of the quasiparticle weight at the Fermi energy, given by Eq. (17), as the magnitude of the Coulomb interactions increase, shown in Fig. 5a. The quasiparticle weight is 1 for the noninteracting case, and it remains 1 in the presence of interactions at the bottom and at the top of the band, since the single electron and the single hole behave as free particles. When the band filling increases from empty or decreases from full the quasiparticle weight decreases in the presence of interactions and is lowest at 
the half filled band. Thus the spectral function will have largest incoherent contribution at and close to half-filling, as one expects in the conventional single band Hubbard model.

The quasiparticle weights in Fig. 5a appear to be electron-hole symmetric. However, a close look reveals that, except for the noninteracting case, the quasiparticle weight for holes is always slightly smaller than the corresponding one for electrons, i.e.

$$
z\left(n_{e}\right)>z\left(2-n_{e}\right)
$$

for $n_{e}<1$. This effect is due to the presence of the second band, and will exist always as long as $\epsilon<\infty$ in our model.

Figure $5 \mathrm{~b}$ shows the effective interaction defined by Eq. (32) versus band filling for these cases. The effective interaction becomes more repulsive as the bare repulsion parameters increase, and is approximately electron-hole symmetric in this case, as in the case of the conventional Hubbard model.

We can estimate the effective mass or the effective hopping amplitude for the quasiparticle from the difference in energy between the ground state and the first excited state. Figure 5c shows the results for these cases. For the noninteracting case $t_{\text {eff }}$ is approximately constant versus band filling, and as the repulsive interactions increase it decreases as the number of carriers in the band increases. Here the electron-hole asymmetry due to the fact that $\epsilon<\infty$ is more apparent, with holes always being heavier than electrons.

Next we consider the effect of decreasing the interband energy separation $\epsilon$ in the presence of Coulomb repulsion. Figure 6 shows results for $\epsilon=10,5,4$ and 2. As $\epsilon$ decreases, the quasiparticle properties become increasingly electron-hole asymmetric. The quasiparticle weight, shown in Fig. 6a, is substantially smaller for holes than for electrons as $\epsilon$ becomes small. Similarly the effective hopping amplitude shown in Fig. 6c decreases as the band filling increases, and holes are much heavier than electrons when $\epsilon$ becomes small. The effective interaction (Fig. 6b) remains repulsive for these parameters for all band fillings.

For sufficiently small $\epsilon$ and not too large $U^{\prime}$ however the effective interaction at the top of the band will become atractive. Figure 7 shows results for $\epsilon=2$ and $U^{\prime}=2$. We also show for comparison the cases $\epsilon=2, U^{\prime}=5$ and $\epsilon=5, U^{\prime}=2$, where the effective interaction is always repulsive: both small $\epsilon$ and small $U^{\prime}$ are required to yield an attractive interaction for holes.

It is interesting to examine the quasiparticle weight and effective hopping amplitude for these cases. For the parameters where $U_{\text {eff }}$ is attractive the quasiparticle weight for a single hole is smallest, and the quasiparticle weight is larger for two holes (Fig 7a). This is in contrast to the cases of repulsive $U_{e f f}$, where the quasiparticle weight for 2 holes is smaller than for 1 hole. Similarly the effective hopping (Figure 7c) for two holes is larger than for one hole when $U_{\text {eff }}$ is attractive, and smaller when $U_{e f f}$ is repulsive. In other words, quasiparticles 'undress', i.e. increase their quasiparticle weight and decrease their effective mass, when they pair.

It is also interesting to examine the expectation value of the kinetic energy operator, Eq. (22). This is shown in Figure 8 for the three parameter sets under consideration. The kinetic energy is lowered both when electrons are added to the empty band and when holes are added to the full band. For the case of attractive $U_{\text {eff } f}$ where the single hole is highly dressed (small $z$ ) and the effective hopping is smallest, the kinetic energy is highest, as one would expect. As Fig. 8 shows, in that case only when a second hole is added to the full band the kinetic energy decreases below twice the value of the single hole kinetic energy. This indicates that pairing of holes is driven by lowering of kinetic energy in this model.

Finally, Figure 9 shows the composition of the quasiparticles, as given by the quantities $u_{k_{F}}, v_{k_{F}}$ in Eq. (18). As $\epsilon$ decreases the quasiparticles occupy predominantly the higher orbital (large $v_{k_{f}}$ ) when the number of electrons in the band increases. In the case where the pairing interaction is attractive the quasiparticle weight for a hole in the system with 2 holes is also dominantly in the higher orbital, because of the large probability for two holes to be on the same site; in contrast, for small $\epsilon$ and larger $U^{\prime}$ when the holes are not paired, $v_{k_{f}}$ is much smaller for the system with two holes because the holes occupy different sites. For the case of large $\epsilon$, the quasiparticle weight is dominantly in the lower orbital for all fillings. These results indicate that a necessary but not sufficient condition for pairing in this model is that parameters are such that there is a large probability for electrons to occupy the higher orbital when the band is close to full.

\section{Hole pairing}

As seen in the previous section, the model can give rise to pairing for carriers near the top of the quasiparticle band with repulsive Coulomb interactions. In this section we examine in which regions of parameter space is the effective interaction between holes attractive, and shed some light on its origin.

Figure 10 shows the dependence of $U_{\text {eff }}$ for two holes at the top of the band on various Hamiltonian parameters. As seen in Fig. 10a, $U_{\text {eff }}$ is attractive when the repulsive interaction in the higher orbital, $U^{\prime}$, is sufficiently small. This is because two electrons at a site will occupy dominantly the higher atomic orbital in the regime where pairing occurs. The energy difference between the two atomic orbitals, $\epsilon$, plays an important role: both for large and for small $\epsilon$ the attraction is suppressed, with $\epsilon \sim 1.5$ yielding the largest range of $U^{\prime}$ where $U_{\text {eff }}$ is attractive. This value of $\epsilon$ is found to be optimal for a wide range of the parameters $U, V$ and $t^{\prime}$, i.e. it is set by the value of the hopping amplitude $t=1$.

In contrast, attraction between holes is favored by a 
large value of the lower orbital repulsion $U$, as seen in Figure 10b (we ignore the small region of very small $U$ where $U_{\text {eff } f}$ is attractive which is presumably unphysical). This is because the attraction requires a large change in the state of the remaining electron when a second electron is removed from the site. If $U$ becomes small, two electrons will occupy the smaller rather than the higher orbital and this effect is lost. Similarly, we find (not shown) that large values of the interorbital Coulomb repulsion $V$ are favorable to pairing: the dependence of $U_{\text {eff }}$ on $V$ is similar to the dependence on $U$ shown in Fig. 10b.

As a function of the interorbital hybridization $t^{\prime}$, pairing will occur when $t^{\prime}$ is not too large, as seen in Fig. 10c. Again, the reason is presumably that the states of an electron in a singly and in a doubly occupied site need to be sufficiently different, which will not happen if the two orbitals are strongly mixed by $t^{\prime}$.

It is interesting to examine the change in kinetic energy when carriers pair. In Figure 11 we plot the difference between twice the kinetic energy of a hole in the filled band and that of two holes in the filled band:

$$
\Delta T=2<T>_{1 \text { hole }}-<T>_{2 \text { holes }}
$$

with the kinetic energy operator given by Eq. (22). It can be seen by comparison with Figure 10 that kinetic energy is always lowered $(\Delta T>0)$ when carriers pair, i.e. in the regime where $U_{\text {eff }}$ is attractive. The condition $\Delta T>0$ is necessary but not sufficient to yield $U_{\text {eff }}<0$. This is because pairing is associated with a decrease in kinetic energy and an increase in potential energy in this model.

\section{E. Comparison with conventional pairing}

In conventional models of superconductivity pairing arises from an effective electron-electron attraction induced by coupling to a boson degree of freedom that does not differentiate between electrons and holes. The resulting effective interaction is electron-hole symmetric, and in such models pairing is driven by lowering of potential rather than kinetic energy. We can describe such a scenario in our model by assuming negative values of the on-site interaction $U$, presumably resulting from integrating out a boson. We also take a very large value of the interorbital spacing $\epsilon$ so as to approach a single band Hubbard model, and compute the effective interaction between 2 holes (which is the same as between 2 electrons) from Eq. (32). Not surprisingly, $U_{\text {eff }}$ for this conventional regime is attractive (repulsive) when $U$ is attractive (repulsive).

It is interesting to compare the behavior of the model in the conventional regime (i.e. attractive Hubbard model regime) with that in the regime discussed in the previous subsection where holes pair, which we call 'dynamic Hubbard' regime. We compute the effective interaction $U_{\text {eff }}$ versus $U^{\prime}$ in the dynamic Hubbard regime and versus $U$ (positive and negative) in the conventional regimen, and in figure 12 we plot various properties as a function of the resulting effective interaction $U_{\text {eff }}$ in both regimes. Figure 12a shows the ratio of quasiparticle weights in the system with two holes and one hole. In the conventional regime, $z_{2} / z_{1}$ is less than 1 both when the effective interaction is attractive and when it is repulsive. In contrast, in the dynamic Hubbard regime the quasiparticle weight for the case of two holes becomes much larger than for a single hole when holes pair. Note however that $z_{2}$ can be bigger than $z_{1}$ even when the effective interaction is still repulsive. In Fig. 12b we show the behavior of the effective hopping amplitude defined from the difference in energy between the ground state and first excited state in the model. In the conventional regime, holes becomes heavier than single particles $\left(t_{2} / t_{1}<1\right)$ when they pair, in contrast in the dynamic Hubbard regime paired holes are much lighter than single holes when pairing occurs. However once again pairs can be lighter than single holes even when the effective interaction is still repulsive. This is of course a finite size effect because in an infinite system for low hole concentration holes would be far from each other if not bound in a pair. Finally, Fig. 12c compares the change in kinetic energy upon pairing. In the conventional regime the kinetic energy increases upon pairing, so that the difference between twice the single hole kinetic energy and the pair kinetic energy is negative, while the kinetic energy decreases strongly upon pairing in the dynamic Hubbard regime.

These results illustrate the qualitative difference in the physics of pairing in these two different regimes. In the regime of dynamic Hubbard physics, pairing is associated with undressing 10], i.e. increase in quasiparticle weight, decrease in quasiparticle mass and lowering of kinetic energy. In the conventional regime, the physics of pairing is exactly opposite, pairing is associated with dressing, i.e. smaller quasiparticle weight, larger quasiparticle mass and increase in kinetic energy for the pair. In summary, upon pairing quasiparticles become more coherent and lighter in the dynamic Hubbard regime and more incoherent and heavier in the conventional regime.

Note that the attractive Hubbard model can describe conventional superconductivity both in the short coherence length regime (large attractive $U$ ) and in the regime where the coherence length is thousands of lattice spacings (small attractive $U$ ). The qualitative contrast that we make here between the physics of that model and the physics of the dynamic Hubbard model applies to both regimes.

\section{OPTICAL CONDUCTIVITY}

We calculate the optical conductivity given by Eq. (20). The total optical spectral weight in the model is related to the expectation value of the kinetic energy operator as given by Eq. (21). We will divide the frequency range into a low frequency range with cutoff $\omega_{m}=2$, 
that defines the low frequency spectral weight $A_{l}$, and denote the remaining spectral weight at higher frequencies by $A_{h}$. The low frequency spectral weight includes the 'intra-band' spectral weight; it also includes some low frequency absorption that is not intra-band when the lower band is close to full and $\epsilon$ is not too large.

Figure 13 shows the dependence of the integrated optical spectral weights on band filling, for three sets of parameters. For Fig. 13(a), with $\epsilon=10$, the absorption is approximately electron-hole symmetric; however even in this case with large $\epsilon$ it can be seen that for holes the intra-band low frequency absorption is somewhat lower and the high frequency absorption is somewhat higher than for electrons. As $\epsilon$ decreases (Fig. 13(b)) and even more so when $U^{\prime}$ also decreases (Fig. 13(c)), the intraband absorption becomes much smaller for holes than for electrons. Note also that for the case of Fig. 13(b) where $U_{\text {eff }}$ is still repulsive between holes the low frequency absorption for 2 holes is only slightly larger than for 1 hole; instead, as $U^{\prime}$ is decreased and $U_{\text {eff }}$ becomes attractive (Fig. 13(c)) the intraband optical absorption for 2 holes becomes more than twice the intraband optical absorption for 1 hole, because optical spectral weight is transfered from high to low frequencies when pairing occurs.

Figure 14 compares the optical conductivity for the nearly empty and the nearly full band, for the cases with $\epsilon=10$ and $\epsilon=2$. For the large $\epsilon$ case, the intraband conductivity (per particle) is only slightly smaller for holes than for electrons. Instead, for $\epsilon=2$ there is a dramatic difference in the optical conductivity for electrons and holes: for holes, the intraband conductivity is very small and most of the optical absorption occurs at higher frequencies.

Next we consider the behavior of the optical conductivity upon doping. For the case of large $\epsilon$, it is similar for electrons and for holes, as seen in Figure 15: the intraband conductivity per carrier decreases slightly with doping, and some spectral weight is added at higher frequencies. For the case of small $\epsilon$, Figure 16, the behavior is similar for electrons but dramatically different for holes: in the latter case, there is a large increase in the low frequency spectral weight for the case of two holes, which is due to the undressing of holes when they pair. Furthermore there is an overall shift of the non-intraband spectral weight at higher frequencies to lower frequencies. Similar behavior is found in other realizations of dynamic Hubbard models 15. Such a transfer of optical spectral weight from high to low frequencies has been seen in high $T_{c}$ cuprates upon hole doping and upon lowering the temperature below the superconducting critical temperature 16, 17, 18, 19, 20.

Finally we show the behavior of optical absorption in the regime of conventional pairing. We choose a large value of the on-site attraction to illustrate the behavior clearly, however the qualitative behavior persists for smaller attractive interaction. The optical absorption is electron-hole symmetric as one would expect, and most of the optical spectral weight is at low frequencies (intraband) in this case for all band fillings. Comparing the case of 1 hole and 2 holes (or 1 electron and 2 electrons) in Fig. 17 (b), it is seen that pairing is associated with a decrease in the low frequency optical spectral weight, i.e. quasiparticles become more dressed when they pair. This is in accordance with the behavior found for the quasiparticle weight and effective hopping in figure 12, and qualitatively different to the behavior in the dynamic Hubbard model regime.

\section{RELATION WITH ATOMIC PHYSICS AND WITH REAL MATERIALS}

For any given atom one can relate the parameters in the site Hamiltonian

$$
\begin{aligned}
H_{i}= & U n_{i \uparrow} n_{i \downarrow}+U^{\prime} n_{i \uparrow}^{\prime} n_{i \downarrow}^{\prime}+\epsilon n_{i}^{\prime}+V n_{i} n_{i}^{\prime} \\
& -t^{\prime} \sum_{\sigma}\left(c_{i \sigma}^{\dagger} c_{i \sigma}^{\prime}+\text { h.c. }\right)
\end{aligned}
$$

to atomic quantities by comparison of properties obtained from it and properties of the electronic states of the atom obtained from quantum chemical calculations. As the simplest example we discuss here qualitatively the relation between the Hamiltonian parameters and electrons in a hydrogenic ion with nuclear charge $Z$ within the Hartree approximation. The difference in energy between an electron in the $1 s$ and $2 s$ atomic orbitals corresponds to the energy difference between the two single particle eigenstates in Eq. (36), namely

$$
\sqrt{\epsilon^{2}+4 t^{\prime 2}} \sim 13.6 \times Z^{2} \times \frac{3}{4}
$$

in $e V$ units here and in what follows. For small $t^{\prime}$ we have approximately

$$
\epsilon \sim 10.2 Z^{2}
$$

We will assume $t^{\prime}$ small in what follows so that the strong coupling analysis is applicable. The repulsion $U$ in the lower orbital corresponds to the repulsion of 2 electrons in the $1 s$ orbital

$$
U=17 Z
$$

In the Hartree approximation the single electron orbital with wavefunction $\varphi \propto e^{-Z r}$ expands upon double occupancy to wavefunction $\bar{\varphi} \propto e^{-\bar{Z} r}$, with

$$
\bar{Z}=Z-\frac{5}{16}
$$

We identify the Coulomb repulsion in the upper orbital, $U^{\prime}$, as the repulsion of two electrons in the Hartree expanded orbital, i.e.

$$
U^{\prime}=17 \bar{Z}=U-5.31
$$


We can estimate the Coulomb repulsion between electrons in the two different orbitals, $V$, by calculating the Coulomb integral for one electron in the $1 s$ orbital and another in the expanded Hartree orbital. This yields (in $\mathrm{eV})$

$$
V=27.2 Z \bar{Z} \frac{Z^{2}+3 Z \bar{Z}+\bar{Z}^{2}}{(Z+\bar{Z})^{3}}
$$

Finally we can estimate the intra-atomic hopping $t^{\prime}$ from the overlap matrix element of the single electron wave function in the doubly and singly occupied sites. In the model, that is approximately

$$
S=<\tilde{\downarrow}\left|c_{\uparrow}^{\prime}\right| \tilde{\downarrow} \downarrow>=t^{\prime}\left(\frac{1}{\epsilon}+\frac{1}{V-U^{\prime}-\epsilon}\right)
$$

and in the Hartree atom it is

$$
S=<\varphi \mid \bar{\varphi}>=\frac{(Z \bar{Z})^{3 / 2}}{((Z+\bar{Z}) / 2)^{3}}
$$

It can be seen that in the atom $U>V>U^{\prime}$ for all $Z$. As $Z$ decreases, all Coulomb repulsions decrease, as well as $\epsilon$ and the overlap $S$. This is the regime favorable for pairing in this model. The Hartree calculation is of course very approximate, and in particular it overestimates the overlap matrix element $S$. Nevertheless it illustrates the basic trend. For orbitals higher than the $1 s$ the energy levels become closer in energy and the effects discussed in this paper should become stronger.

In summary, the atomic charge $Z$, with $Z-2$ the charge of the ion when the relevant band is full, is the key atomic parameter. For small $Z$ the parameters in the Hamiltonian studied in this paper move towards the regime of interest, namely small Coulomb repulsion $U^{\prime}$, small interband separation $\epsilon$ and small overlap matrix element $S$. In that regime electron-hole asymmetry in the band becomes dominant, holes become heavily dressed in the normal state and they strongly undress when they pair.

For high $T_{c}$ cuprates the relevant band of interest is one formed by overlapping planar oxygen $p \pi$ orbitals in the $\mathrm{CuO}_{2}$ planes 21. Since in the undoped system (no holes) the ion is $O^{=}, Z=0$ in this case. For $M g B_{2}$, the relevant band is formed by overlapping boron $p_{x y}$ orbitals in the $B^{-}$planes 22], and $Z=1$. The fact that the planes are negatively charged in both cases $(Z<2)$ favors the physics discussed here, with the effects stronger for the cuprates due to the smaller $Z$. Even stronger hole dressing and higher $T_{c}$ 's would be expected in a structure with even smaller $Z$, for example if one managed to make a material with $N \equiv$ planes doped with some holes $(Z=$ $-1)$.

The material $L i B C$ has been recently proposed as a candidate for high temperature superconductivity when hole-doped, by analogy with $M g B_{2}$, within electronphonon theory 23]. Because the $(B C)^{-}$planes in that material would be less negatively charged than the $\left(B_{2}\right)=$ in $M g B_{2}$, i.e. effectively $Z=1.5$ instead of $Z=1$, we expect this not to be a modification of $M g B_{2}$ conducive to higher $T_{c}$ 's within the physics discussed here. If such material was found to have a $T_{c}$ larger than $M g B_{2}$, as predicted [23], it would directly contradict the assumptions of this paper and prove the inapplicability of the concepts discussed here to real materials.

\section{DISCUSSION}

Electrons in solids interact with each other with an interaction strength $\left(e^{2}=14.4 \mathrm{eVA}\right)$ that is of the same magnitude as the interaction strength of electrons with ions. It was recognized from the beginnings of solid state physics that Bloch's approach of prioritizing the electronion interaction over the electron-electron interaction was an ad-hoc assumption that could certainly not be rigurously justified. Even though Landau's Fermi liquid theory with the concept of a quasiparticle provides an explanation for the fact that many properties of solids look amazingly 'independent-electron-like', the fundamental role of electron-electron interactions in solids is still not well understood.

This paper is part of a continuing effort to understand the role of electronic correlation in energy bands. We argue that a key fact that has been ignored in previous treatments of the problem is the dependence of quasiparticle weight on band filling and the fundamental role of electron-hole asymmetry. In this paper we studied a 'minimal model' that incorporates these key features. We believe that this physics is part of the physics of all electronic energy bands: that quasiparticle weights at the Fermi level when the band filling $n(0<n<2)$ is below and above the half-filled band $(n=1)$ are related by

$$
z(n)>z(2-n)
$$

with $n<1$, i.e. that holes are always more dressed than electrons. How different the quasiparticle weights in the lower and upper halfs of the band are determines how important the new physics originating in this effect is. This in turn depends on the ionic charge $Z(Z-2=$ ionic charge when the band is full) with the strongest effects occurring for small $Z$. Because quite generally in an atom the intra-orbital Coulomb repulsion is linear in $Z$ (e.g. Eq. (39)) and the energy level spacing is quadratic in $Z$ (e.g. Eq. (38)) the effects discussed here will become unimportant for sufficiently large $Z$.

We have called the models describing this physics 'dynamic Hubbard models'. In these models, unlike the case of the conventional Hubbard model, the strength of the on-site repulsion $U$ becomes a dynamical variable that can take more than one value depending on the state of the two electrons in the atom. Here this dynamics is incorporated by having two electronic orbitals per site; in other work we have described this dynamics with a single electronic orbital per site and an auxiliary boson degree of freedom [13, 15, 24, 25]. While the model discussed here is more realistic and closer to the physics of real atoms, the models with auxiliary boson degrees of 
freedom are simpler to treat theoreticaly and thus may yield useful insight into the fundamental physics of this class of models. From the results in this paper and in previous work we believe that dynamic Hubbard models with only electronic degrees of freedom and those with auxiliary boson degrees of freedom share the same fundamental physics.

We have studied the two-orbital model by exact diagonalization of a small cluster. It should be possible to study larger clusters with more computing power and more sophisticated numerical techniques such as Lanczos diagonalization, density-matrix renormalization group and quantum Monte Carlo. We believe that the qualitative physics found here is likely to exist in larger systems.

The calculations in this paper yield the properties of interacting electrons in a model Hamiltonian for the entire range of band fillings from empty to full, without uncontrolled approximations. Before this work such studies had only been performed for simpler models such as the single band conventional Hubbard model, which as we have argued lacks some essential physics. The results found here should qualitatively apply to all electronic energy bands in solids.

The results found here corroborate some of our earlier findings concerning the importance of electron-hole asymmetry 10 and display clearly the interpolation between the conventional understanding of electronic correlations in energy bands and the physics stressed in the theory of hole superconductivity. In the conventional understanding electrons and holes are similar, quasiparticles are undressed when the band is almost empty and almost full, and the dressing and importance of electronelectron interactions increases as one approaches the halffilled band from either side. Instead, in the theory of hole superconductivity in its simplest interpretation the dressing of a quasiparticle increases monotonically as the band filling increases from the empty to the full band. As we have seen in this paper, the actual situation is always in-between these two limiting descriptions, with the relationship Eq. (45) holding in all cases.

The essential difference between conventional (static) and dynamic Hubbard models concerning 'intra-band' physics is that the state of a given electron is the same in the singly and doubly occupied atom in the static Hubbard model, while it is different in the dynamic Hubbard model. Through this modification of the state the intraband bare particles, which were strongly interacting with repulsion $U$, become weakly interacting quasiparticles with interaction $U^{\prime}$. This occurs at the level of a single site, and is expressed by the relation between bare particle operators $c_{i \sigma}$ and quasiparticle operators $\tilde{c}_{i \sigma}$

$$
c_{i \sigma}=\left[1+(S-1) \tilde{n}_{i,-\sigma}\right] \tilde{c}_{i \sigma}
$$

The quasiparticle dynamics is described by the kinetic energy Eq. (30) and the local repulsion $U^{\prime}$, and their weight is further modified by the weak interactions in the quasiparticle band. The quasiparticle weight at the Fermi energy can be approximately written as

$$
z(n)=\left[1+(S-1) \frac{n}{2}\right]^{2} z_{i b}(n)
$$

with $0 \leq n \leq 2$ the band filling, and the 'intraband' quasiparticle weight $z_{i b}$ defined by

$$
z_{i b}=\left|<0_{N-1}\right| \tilde{c}_{k_{F} \sigma}\left|0_{N}>\right|^{2}
$$

calculated using the ground states of the Hamiltonian for the intraband quasiparticles (Eq. (30) plus weak on-site repulsion). In particular $z_{i b}(n \rightarrow 0)=z_{i b}(n \rightarrow 2)=1$ and is smallest near the half-filled band, as in the conventional Hubbard model. The factor multiplying $z_{i b}$ in Eq. (47) isolates the main effect of electron-hole asymmetry. However even $z_{i b}$ will exhibit some additional electronhole asymmetry (of the same sign) due to the dependence of the effective bandwidth on filling Eq. (33): the residual intraband interactions will more strongly dress the quasiparticles in the upper half of the band where the effective bandwidth is smaller.

For materials with large ionic charge $Z, S$ will be close to 1 , electrons and holes will be very similar and the dominant dressing will occur near the half-filled band. Instead, for materials with small $Z, S$ will be much smaller than 1 , the physics of hole superconductivity will dominate, holes will be highly dressed near the full band and strongly undress as the local hole concentration increases. We propose that the physics of high temperature superconductivity in solids is described by the latter regime. As the parameters become less extreme with increasing ionic charge $Z$ the dressing of holes in the normal state becomes less extreme, the undressing effect of pairing becomes less apparent, the coherence length of the Cooper pairs increases and one moves towards the regime of 'conventional' superconductivity 26].

\section{Acknowledgments}

The author is grateful to Fred Driscoll for providing the computer facilities where this work was performed. This work was NOT supported by the National Science Foundation.
[1] J.C. Slater, "Quantum Theory of Atomic Structure", Mc Graw Hill, New York, 1960.
[2] J. Hubbard, Proc.Roy.Soc. London A276, 238 (1963). 
FIG. 1: Non-interacting band structure for the model under consideration for various parameters. (a) As $\epsilon$ increases, the gap between lower and upper band increases. (b) As $t^{\prime}$ increases the bandwidth of the lower band increases and that of the upper band decreases.

[3] J.E. Hirsch, Phys.Rev.B 65, 184502 (2002) and references therein.

[4] L.M. Roth, Phys.Rev. 149, 306 (1966); K.I. Kugel and D.I. Khomskii, Sov.Phys. JETP 37, 725 (1973); M.Cyrot and C. Lyon Caen, J. Phys. (Paris) 36, 253 (1975); W. Gill and D.J. Scalapino, Phys.Rev.B 35, 215 (1987); K. Kuei and R.T. Scalettar, Phys.Rev.B 55, 14968 (1997).

[5] L.M. Falicov and J.C. Kimball, Phys. Rev. Lett. 22, 997 (1969); Q.Si, G. Kotliar and A. Georges, Phys. Rev. B 46, 1261 (1992); P. Farkasovsky, Phys. Rev. B 51, 1507 (1995); T. Portengen, T. Ostreich and L.J. Sham, Phys. Rev. B 54, 17452 (1996); J.K. Freericks and V. Zlatic, Phys. Rev. B 58, 322 (1998).

[6] J.M. Robinson, Phys.Rep. 51, 1 (1979); P. Schlottmann, Phys. Rev. B 22,613 (1980); B.H. Brandow, Phys. Rev. B 33,215 (1986); V.Z. Vulovic and E. Abrahams, Phys. Rev. B 36,2614(1987); A. Houghton, N. Read and H. Wu, Phys. Rev. B 33, 3782 (1988); H. Schweitzer and G. Czycholl, Sol.St.Comm. 74, 735 (1990).

[7] J.E. Hirsch, Phys.Rev. B 43, 11400 (1991).

[8] K. Held, C. Huscroft, R. T. Scalettar and A. K. McMahan, Phys.Rev.Lett. 85, 373 (2000).

[9] P. Maldague, Phys. Rev. B 16, 2437 (1977).
[10] J.E. Hirsch, Phys. Rev. B 62, 14487 (2000), 62 , 14498 (2000), and references therein.

[11] J. E. Hirsch and F. Marsiglio, Phys. Rev. B 62, 15131 (2000) and references therein.

[12] R.M. Fye, M.J. Martins and R.T. Scalettar, Phys. Rev. B 42, 6809 (1990).

[13] J.E. Hirsch, Phys.Rev. B 65, 214510 (2002).

[14] J. Wagner, W. Hanke and D.J. Scalapino, Phys.Rev. B 43, 10517 (1991).

[15] J.E. Hirsch, cond-mat/0205006 (2002).

[16] S.Uchida, T.Ido, H.Takagi, T. Arima, Y. Tokura and S. Tajima, Phys. Rev. B 43, 7942 (1991).

[17] H. J. A. Molegraaf, C. Presura, D. van der Marel, P. H. Kes, and M. Li Science 295, 2239 (2002).

[18] A.F. Santander-Syro et al, cond-mat/0111539 (2001).

[19] D.N. Basov et al, Science 283, 49 (1999).

[20] F. Gao, D.B. Romero, D.B. Tanner, J. Talvacchio and M.G. Forrester, Phys.Rev. B 47, 1036 (1993).

[21] J. E. Hirsch and F. Marsiglio, Phys.Rev. B 43, 424 (1991).

[22] J. Kortus, I. I. Mazin, K. D. Belashchenko, V. P. Antropov, and L. L. Boyer, Phys.Rev.Lett. 86, 4656 (2001).

[23] H. Rosner, A. Kitaigorodsky and W.E. Pickett, Phys. Rev. Lett. 88, 127001 (2002).

[24] J.E. Hirsch, Phys. Rev. Lett. 87, 206402 (2001).

[25] J.E. Hirsch, Phys.Lett. A 134, 451 (1989).

[26] "Superconductivity", ed. by R.D. Parks, Marcel Dekker, New York, 1969. 
FIG. 2: Schematic depiction of states in 4-site cluster for different band filling. Because of the large $U$ in the lower orbital, two electrons at the same site will predominantly occupy the higher orbital. The number of states in the Hilbert space for each filling is also given.

FIG. 3: Effective interaction Eq. (32) versus band filling for different boundary conditions, for four sets of parameters. $t=1$ here and in the following figures. For all cases here $U=10$ and $V=6$. The lines through the data are a guide to the eye. Solid line: $U^{\prime}=2, \epsilon=2, t^{\prime}=0.2$; dashed line: $U^{\prime}=$ $4, \epsilon=2, t^{\prime}=0.2$; dash-dotted line: $U^{\prime}=2, \epsilon=1, t^{\prime}=0.2$; dotted line: $U^{\prime}=2, \epsilon=2, t^{\prime}=0.5$.

FIG. 4: Energy eigenvalues for the different band fillings. Parameters are: (a) $\epsilon=12, t^{\prime}=0.2, U=V=U^{\prime}=0$; (b) $\epsilon=6, t^{\prime}=0.2, U=20, V=12, U^{\prime}=2$. The dashed lines show the separation between the 'intra-band' states described approximately by the Hamiltonian Eq. (3) below it, and the rest of the states in the Hilbert space. For other less extreme parameters no clear separation is seen. Note that in the interacting case (b) the spectrum is strongly electronhole asymmetric, with the lower band bandwidth for holes $\left(N_{e}>4\right)$ much smaller than for electrons $\left(N_{e}<4\right)$.

FIG. 5: The parameters used are $\epsilon=10, t^{\prime}=0.2$ and the following interactions: solid lines: $U=V=U^{\prime}=0$; dashed lines: $U=3, V=2, U^{\prime}=1$; dash-dotted lines: $U=6, V=4, U^{\prime}=3$; dotted lines: $U=10, V=6, U^{\prime}=5$. Plotted versus bandfilling $N_{e}$ are (a) quasiparticle weight at the Fermi energy, Eq. (17); (b) effective interaction Eq. (32); (c) effective hopping defined as the energy gap between the ground state and the first excited state. Note that even for this case of large $\epsilon$ there is a small electron-hole asymmetry, with the quasiparticle weight and effective hopping being slightly smaller for holes than for electrons.

FIG. 6: Same as figure 5 for $t^{\prime}=0.2, U=10, V=6, U^{\prime}=5$ and $\epsilon$ values given in the figures. As $\epsilon$ decreases holes become more incoherent and heavier, i.e. smaller $z$ and $t_{\text {eff }}$.

FIG. 7: Same as figure 5 for $t^{\prime}=0.2, U=10, V=6$ and values of $\epsilon$ and $U^{\prime}$ given in the figure. For small $\epsilon$ and $U^{\prime}$ (solid lines) the effective interaction is attractive for holes; in that case, the quasiparticle weight at the Fermi energy for the band filled with 2 holes is larger than for the band with 1 hole (a), and the effective hopping is larger for the band with 2 holes than for the band with 1 hole.

FIG. 8: Expectation value of the kinetic operator Eq. (22) for the same parameters as in Fig. 7. Note that for the case of attractive effective interaction (full line) the kinetic energy for two holes is lower than twice the kinetic energy for one hole, while in the cases of repulsive effective interaction it is higher. 
FIG. 9: Composition of the quasiparticles, from Eq. (18), for the parameters of Fig. 7. $u_{k_{F}}$ and $v_{k_{F}}$ give the amplitude of the quasiparticle at the Fermi energy in the lower and upper atomic orbital. As the band filling increases $u_{k_{F}}$ decreases and $v_{k_{F}}$ increases, with the changes being largest for the case of attractive effective interaction

FIG. 10: Dependence of effective interaction for two holes in the filled band on Hamiltonian parameters. The values of $\epsilon$ are given in the figures. (a) Versus $U^{\prime}$, with $U=10, V=$ $6, t^{\prime}=0.2$; (b) versus $U$, with $U^{\prime}=2, V=6, t^{\prime}=0.2$; (c) versus $t^{\prime}$, with $U=10, V=6, U^{\prime}=2$.

FIG. 11: Dependence of kinetic energy difference between single holes and pair of holes, Eq. (35), on Hamiltonian parameters, for the same cases as Fig. 9.

FIG. 12: Comparison of behavior of model in conventional (electron-hole symmetric) regime and regime of dynamic Hubbard physics. Parameters used for dynamic Hubbard regime are $U=10, V=6, \epsilon=2, t^{\prime}=0.2$ and $U^{\prime}$ ranging from 0 to 6 ; for conventional regime, $\epsilon=100, t^{\prime}=0.2, V=6, U^{\prime}=5$ and $U$ ranging from -1.5 to 8 . In both cases $U_{\text {eff }}$ is calculated from Eq. (32) and the results are plotted versus $U_{\text {eff }}$. (a) Ratio of quasiparticle weights for two holes and for one hole; (b) ratio of hopping amplitudes for two holes and one hole; (c) difference in kinetic energy of single holes and paired holes, Eq. (35).

FIG. 13: Integrals of optical conductivity. $A_{l}$ denotes the low frequency integral Eq. (23), with cutoff $\omega_{m}=2 ; A_{h}$ denotes the high frequency optical spectral weight for $\omega>\omega_{m}$, and $A=A_{l}+A_{h}$ the total optical spectral weight Eq. (21). Parameters are $U=10, V=6, t^{\prime}=0.2$ and values of $U^{\prime}$ and $\epsilon$ given in the figures (same parameters as Figs. 7-9).

FIG. 14: Comparison of optical conductivity for one electron and one hole for $t^{\prime}=0.2$ and values of $\epsilon$ given in the figure. Here and in the following figures of optical conductivity, the $\delta$ functions in Eq. (20) are broadened to Lorentzians with width $\Gamma=0.25$. The lowest frequency $\delta$-function at the 'Drude precursor' frequency 14 is shifted to $\omega=0$ and represented by a Drude form (semi-Lorentzian). Note that for large $\epsilon$ the conductivities for electrons and holes are very similar, for small $\epsilon$ the conductivity for holes is very small and in particular the intra-band conductivity is much smaller than for the case of large $\epsilon$.

FIG. 15: Comparison of optical conductivity for 1 carrier and 2 carriers in the band. The optical conductivity for one carrier is multiplied by a factor 2 to make it comparable to the optical conductivity for two carriers. (a) electrons, (b) holes. Here and in the next figure $U=10, V=6, t^{\prime}=0.2$. Here, $\epsilon=$ $10, U^{\prime}=5$. Note that the optical conductivity is similar for 1 and 2 carriers (normalized to number of carriers) both for electrons and for holes, with the low frequency absorption (per carrier) being slightly smaller when the number of carriers is larger. 
FIG. 16: Same as Fig. 15 for parameters $\epsilon=2, U^{\prime}=2$. For the case of electrons the results are similar to Fig. 15, for the case of holes they are very different: for the case of 2 holes there is a large increase in low frequency absorption and an overall shift in optical absorption to lower frequencies.

FIG. 17: Optical absorption for parameters in the 'conventional' regime, given in the caption of Fig. 12, with strong on-site attractive interaction $U=-8$, giving rise to effective attraction $U_{e f f}=-5.6$. (a) Integrated optical absorption, same cutoff as in Fig. 13. Note that the low frequency absorption does not increase as the number of carriers increases from 1 to 2. (b) Optical conductivity for 1 and 2 holes (essentially the same as for 1 and 2 electrons for these parameters). Note that upon pairing the low frequency absorption decreases strongly as paired carriers are more highly dressed. 

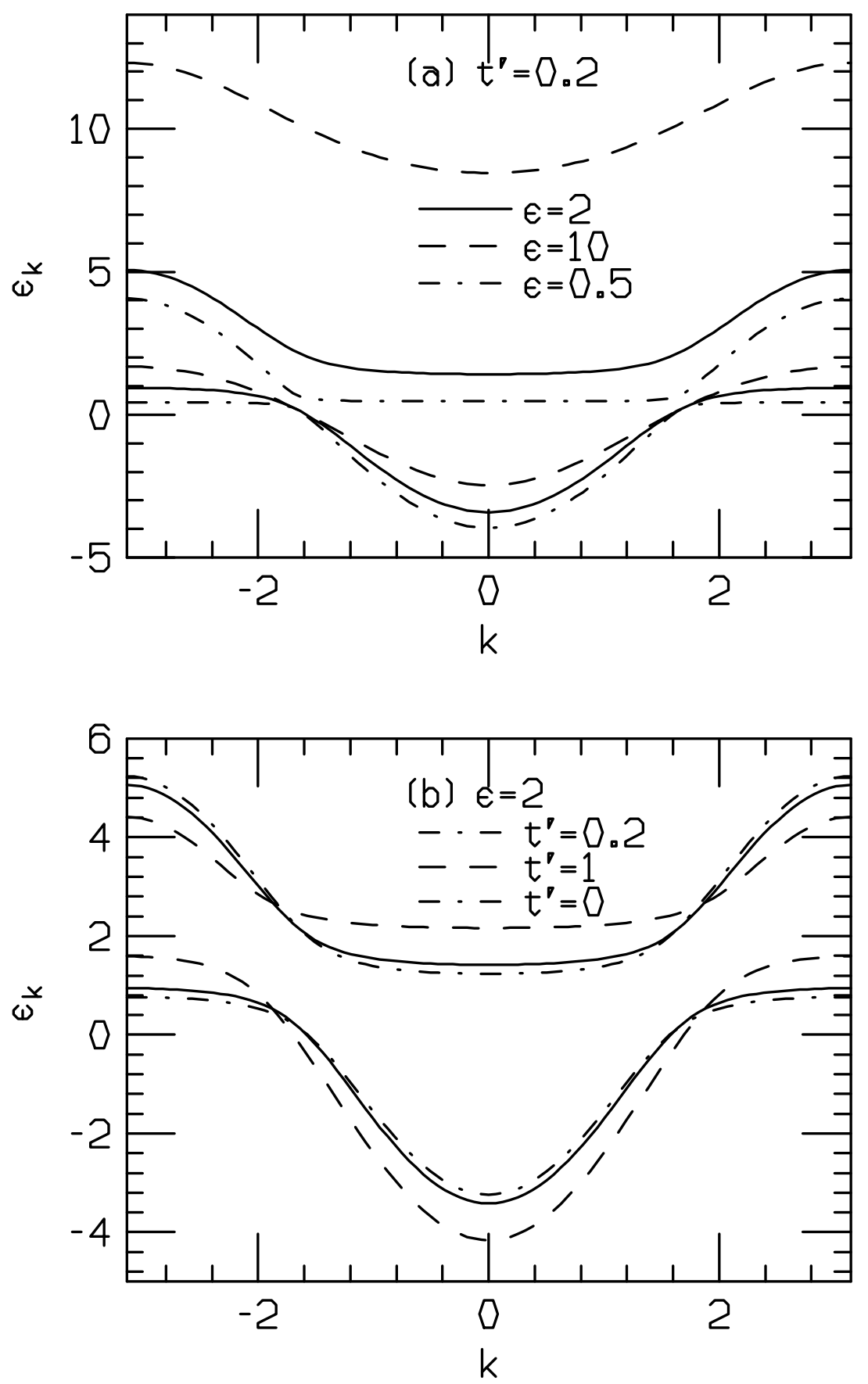

Figure 1 


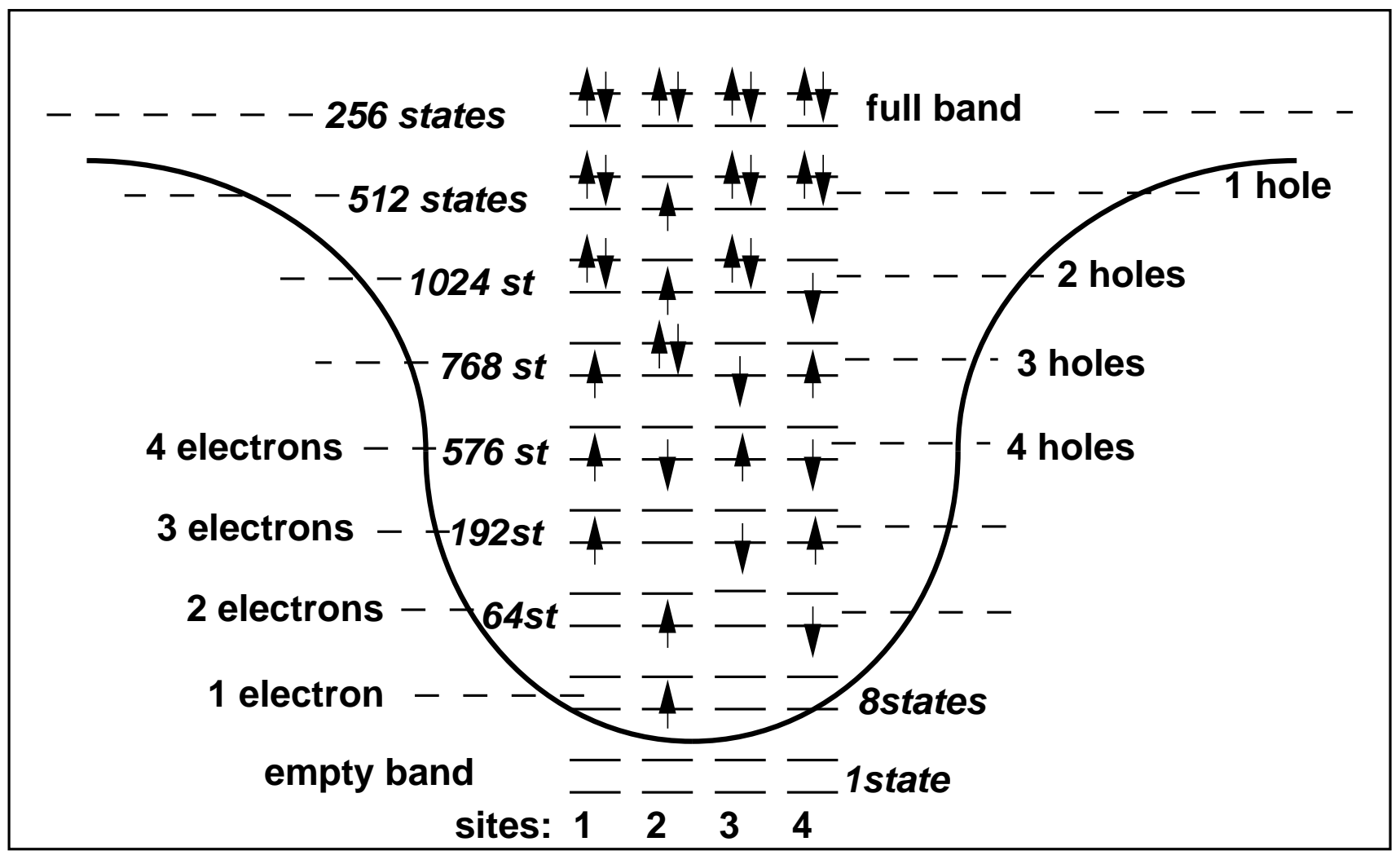

Figure 2 

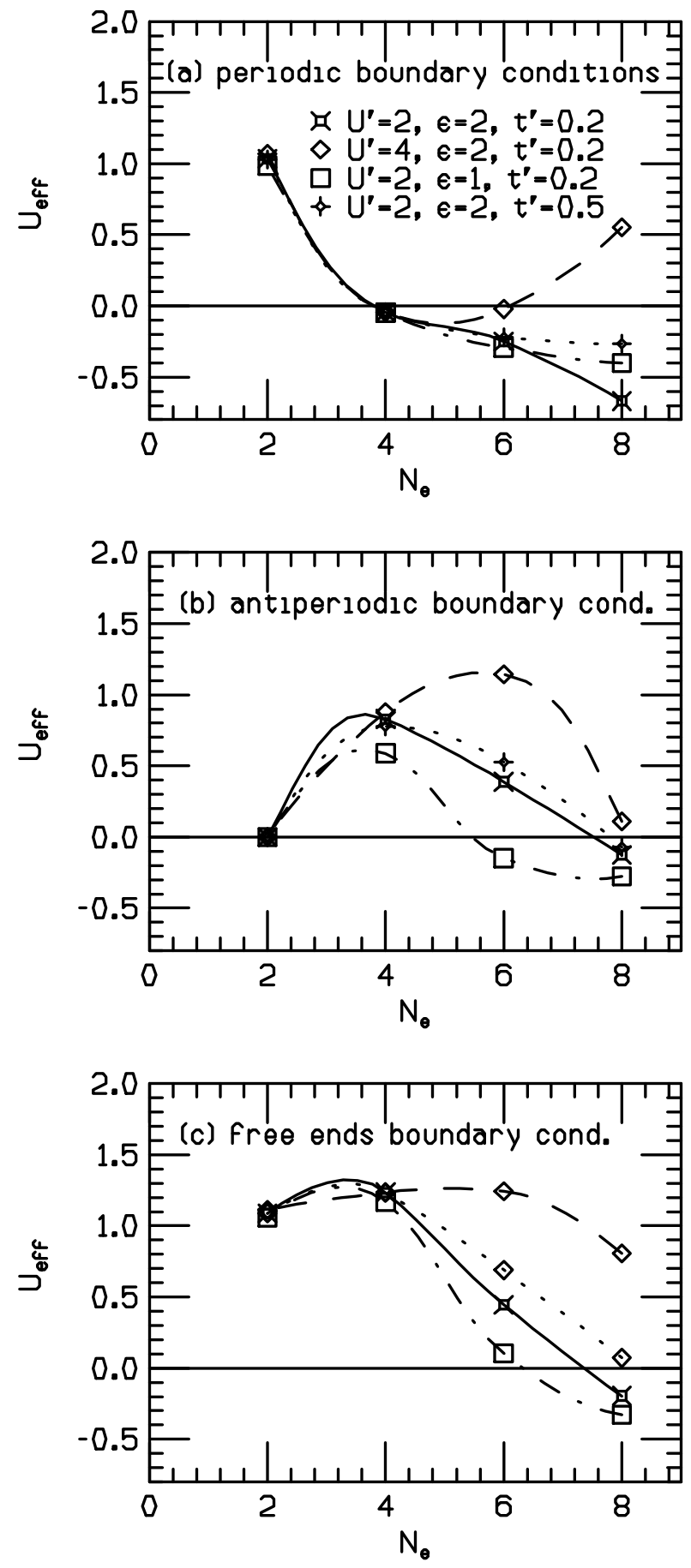

Figure 3 

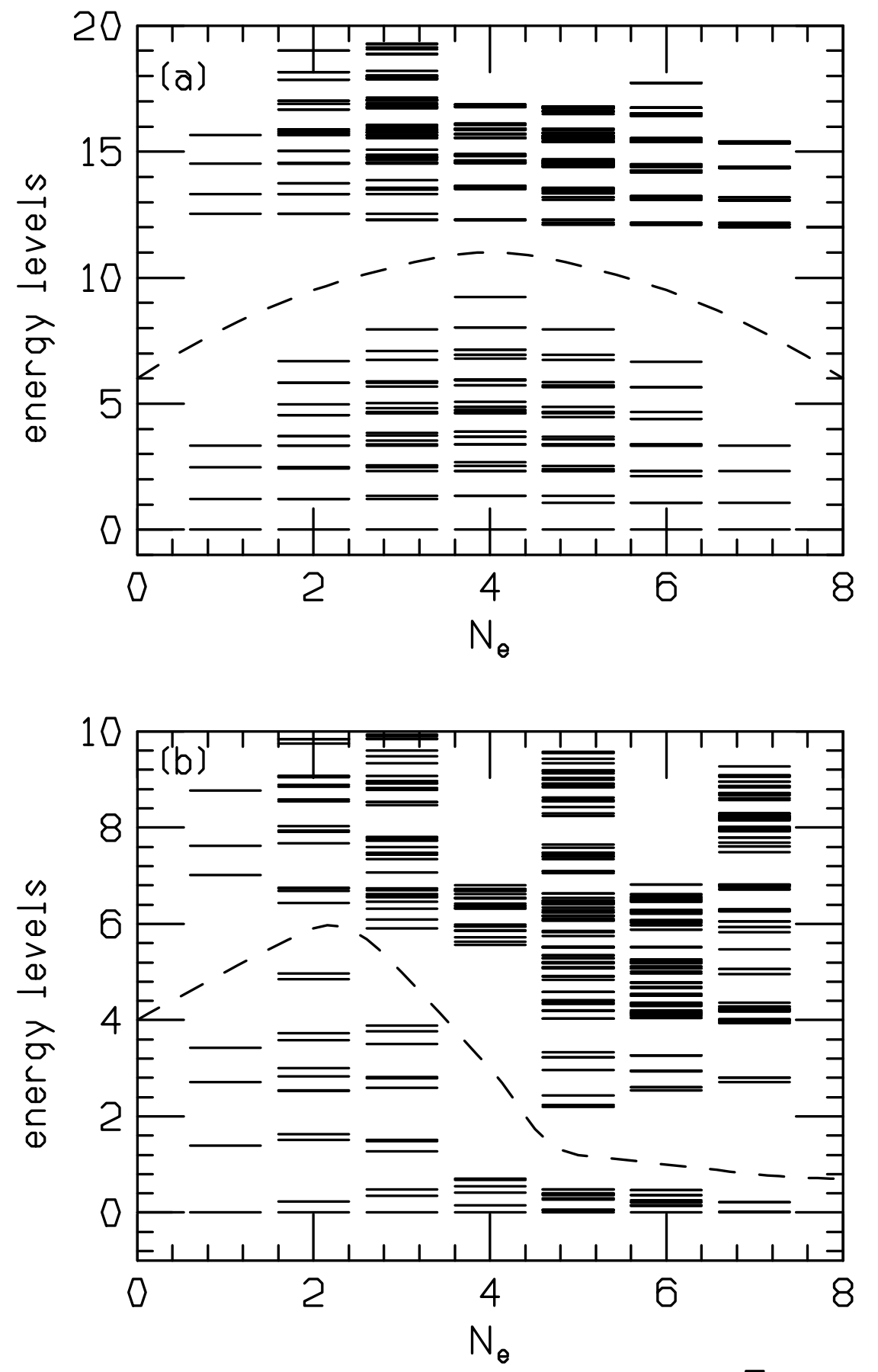

Figure 4 

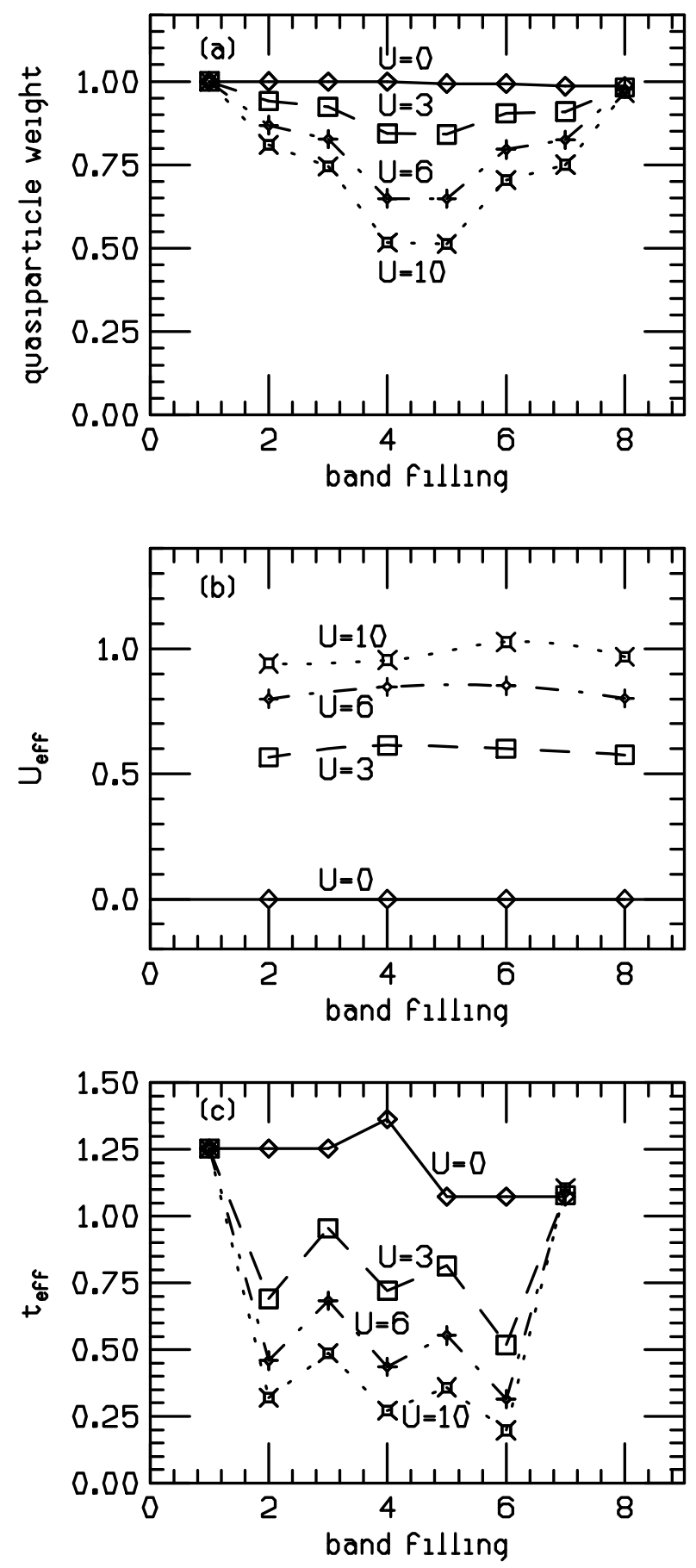

Figure 5 

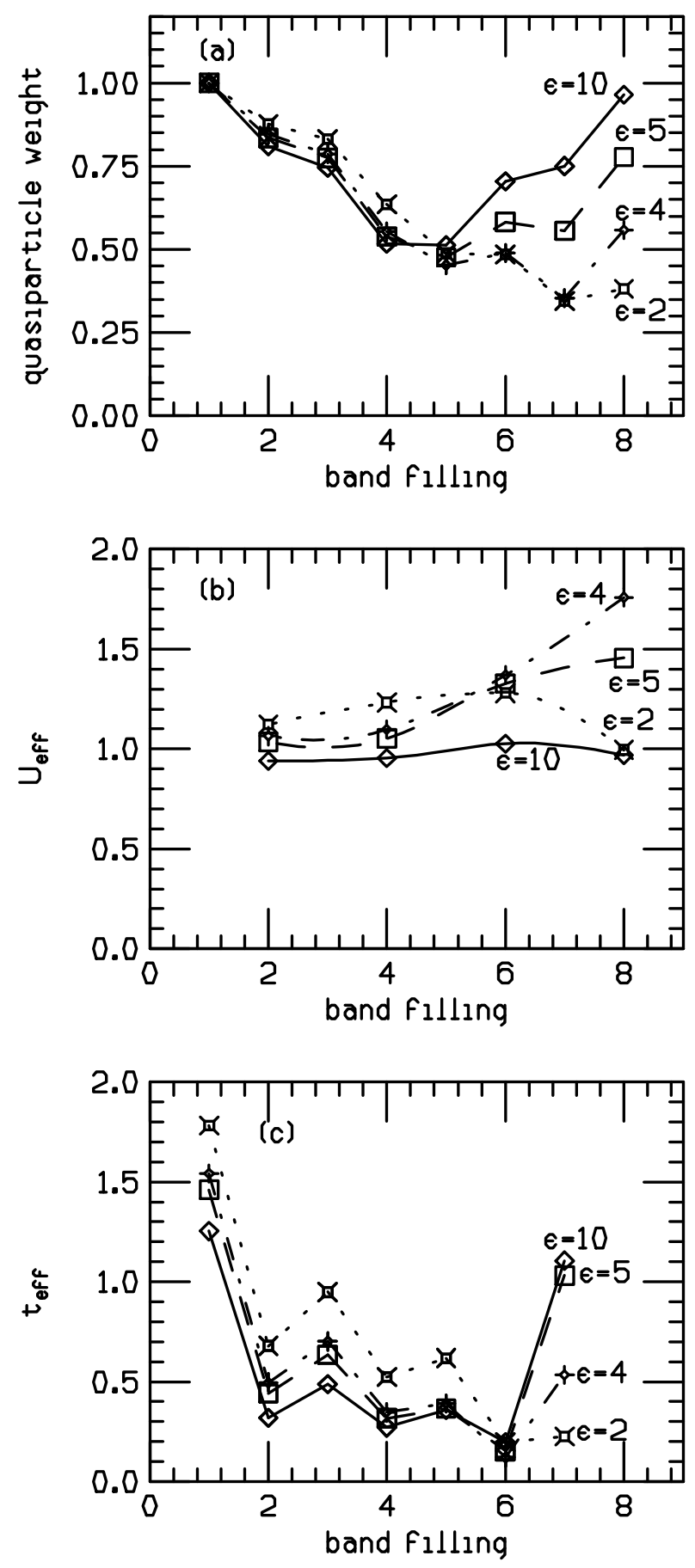

Figure 6 

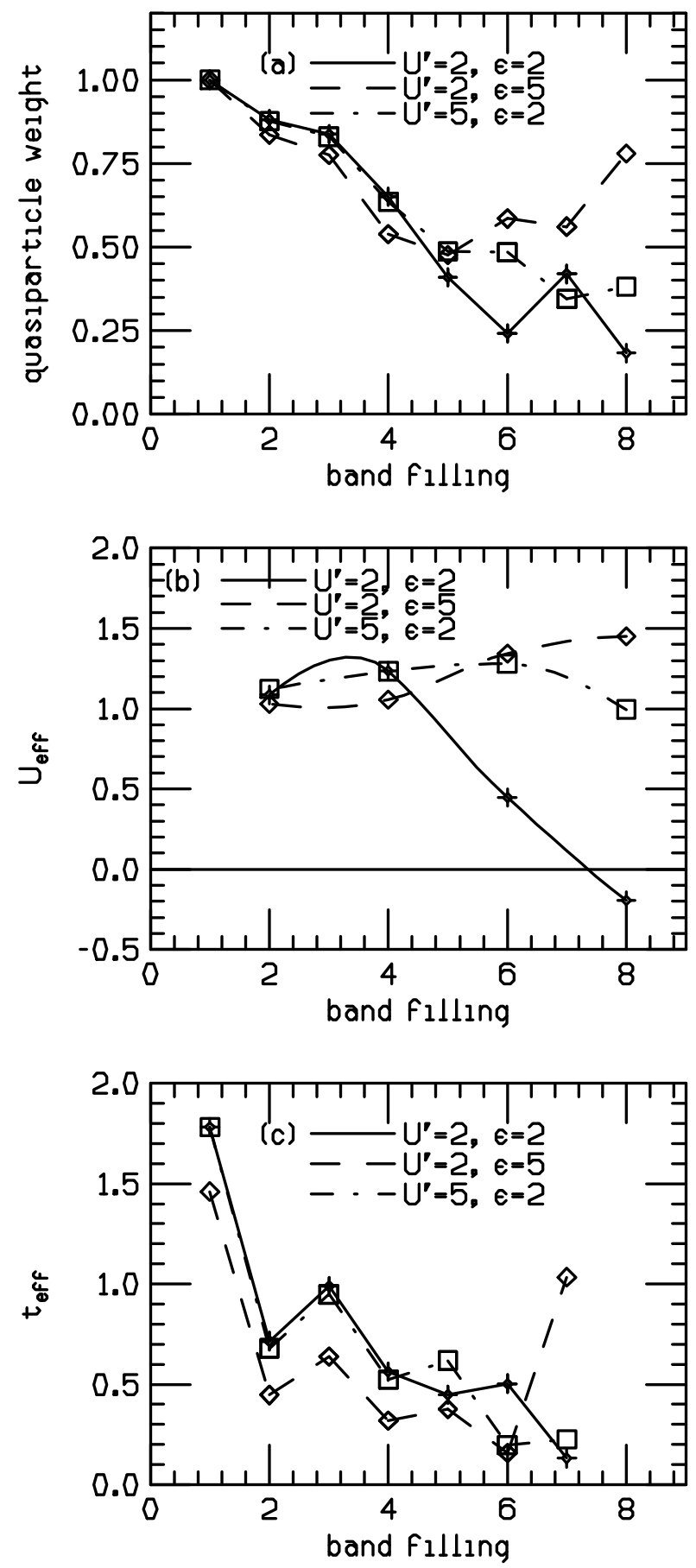

Figure 7 


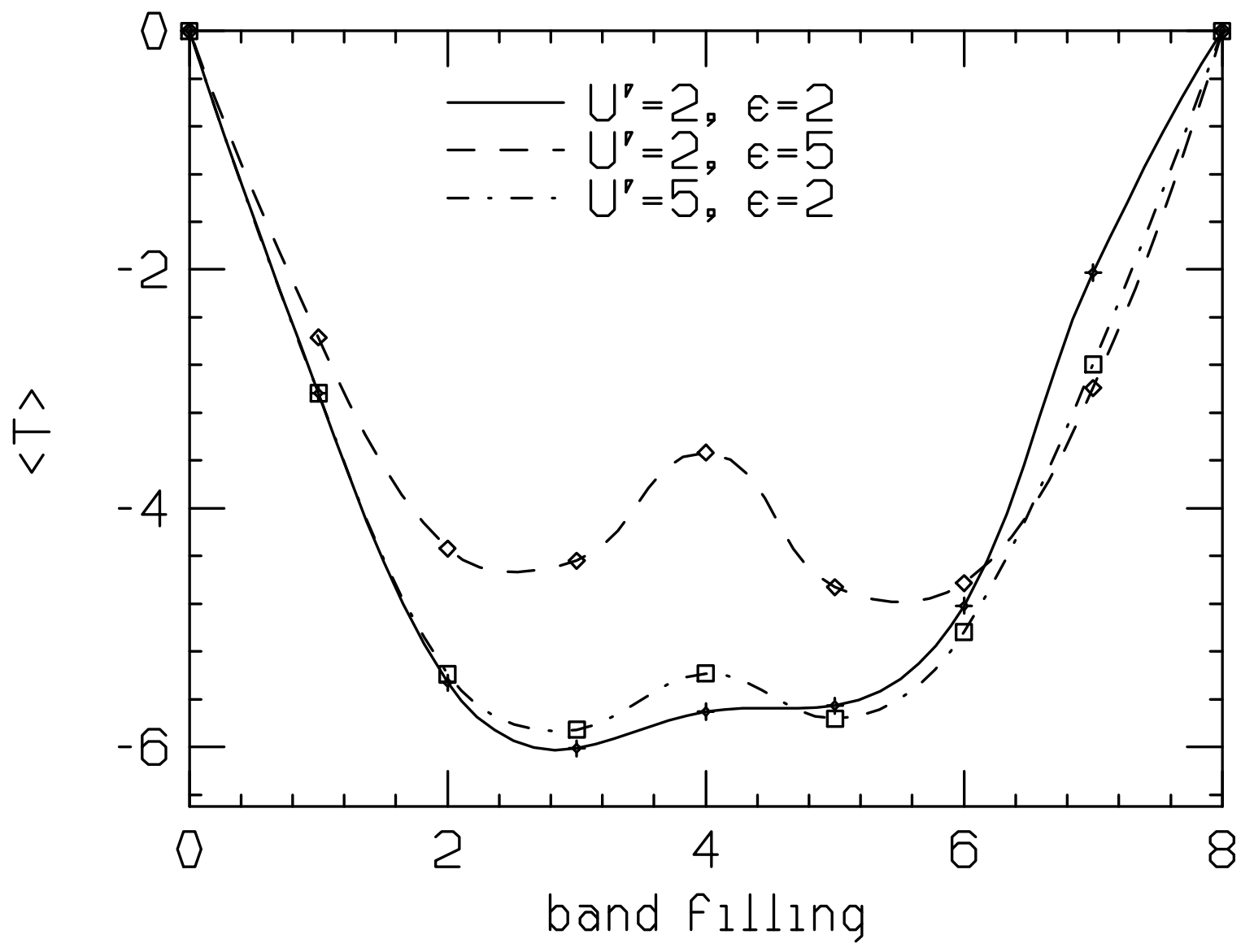

Figure 8 


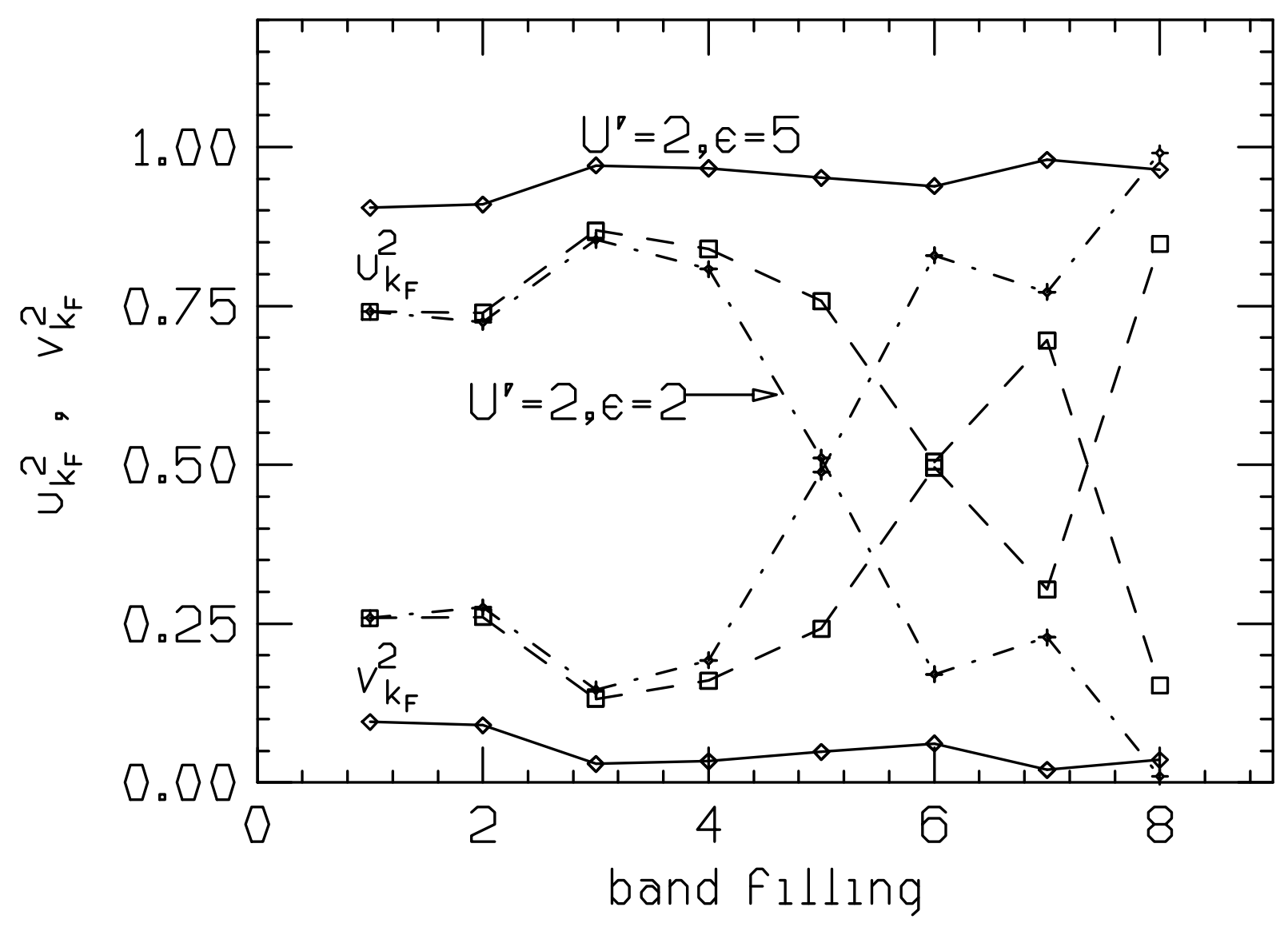

Figure 9 

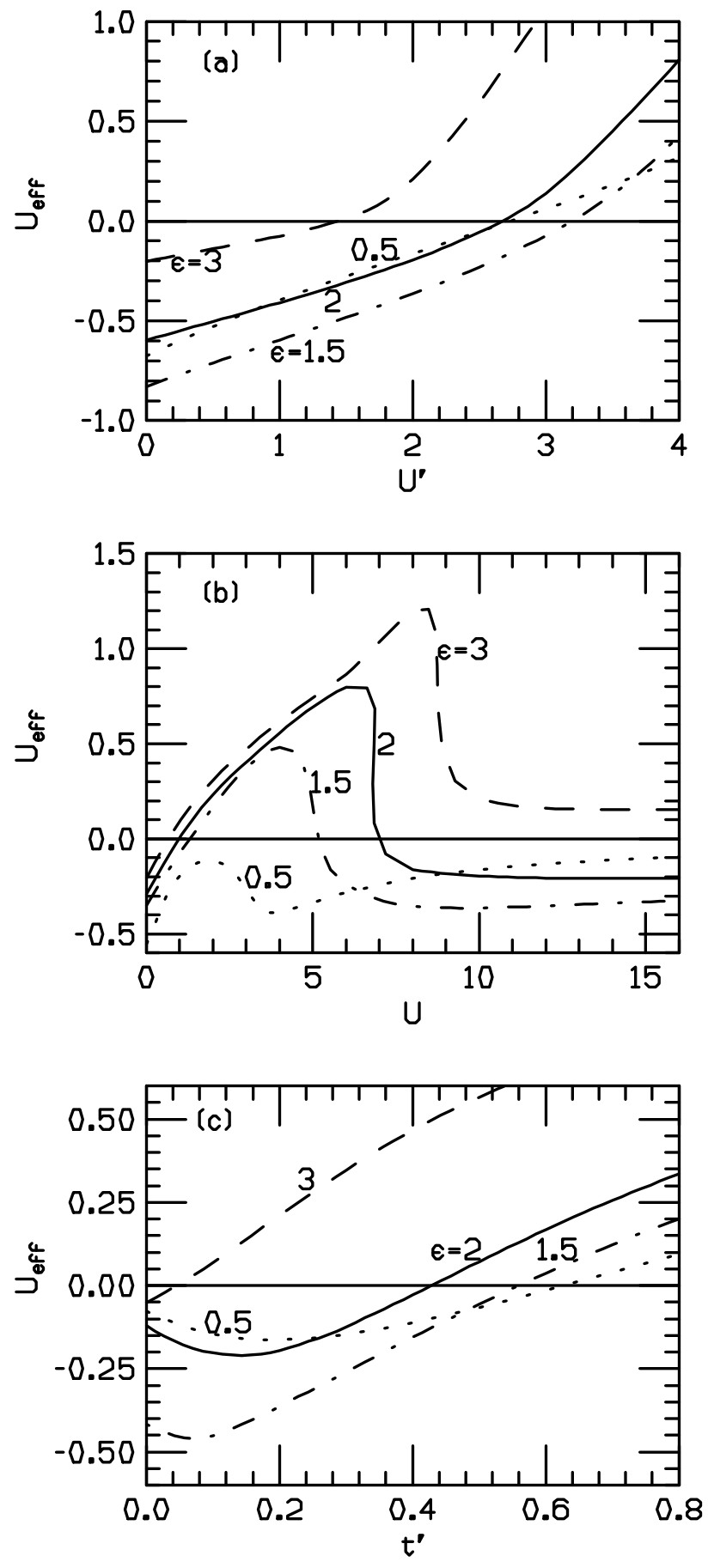

Figure 10 


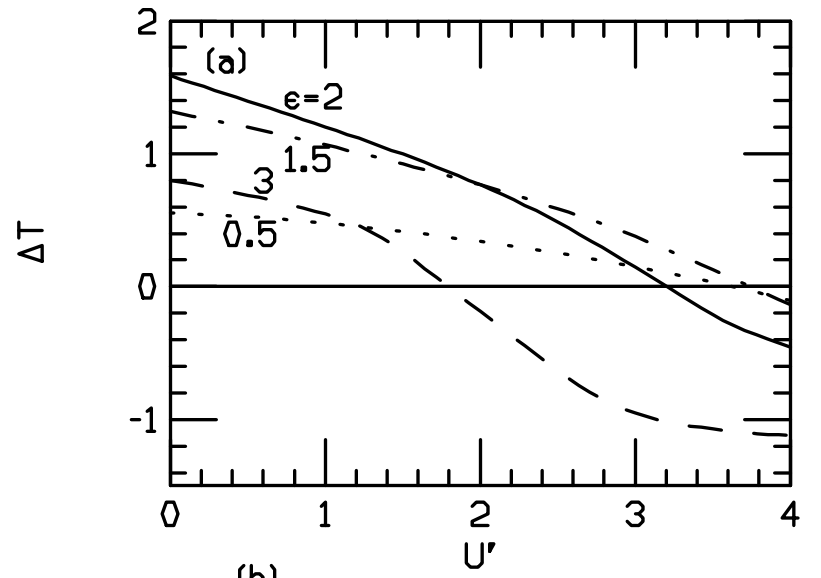

(b)
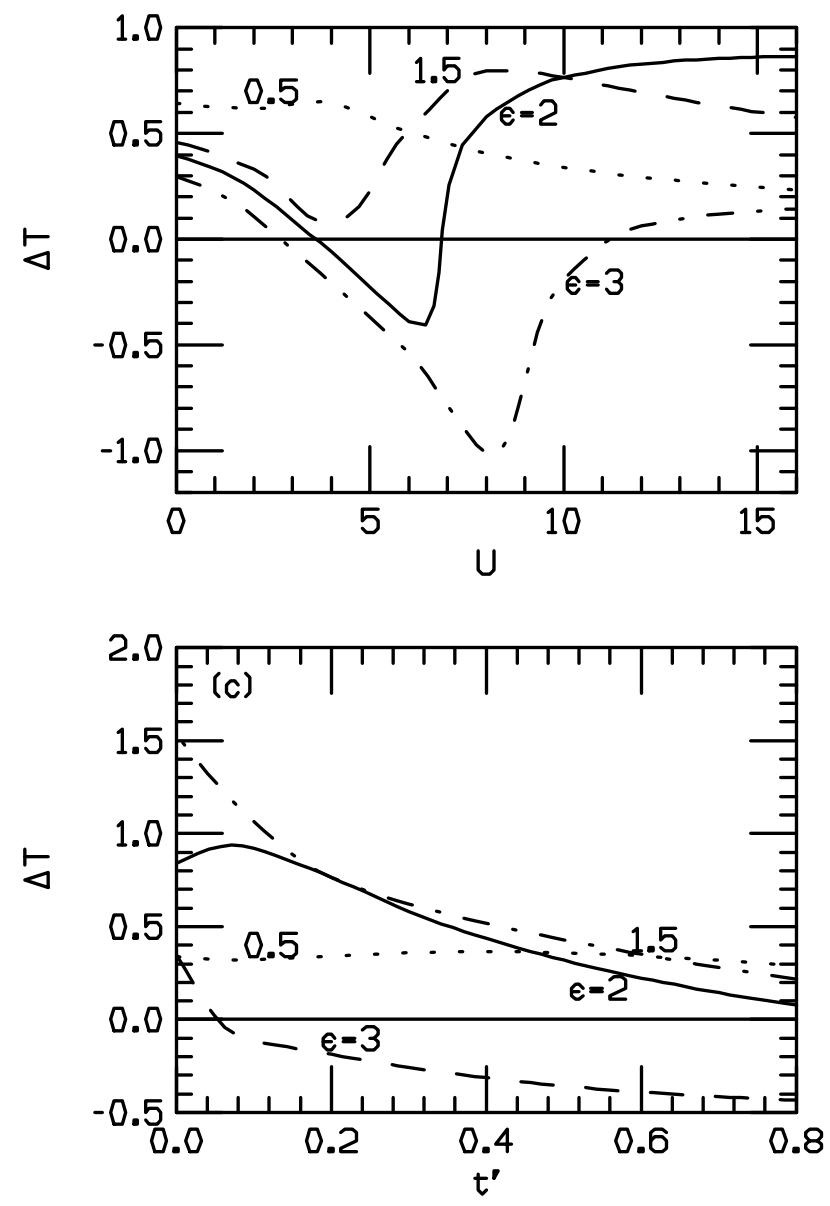

Figure 11 

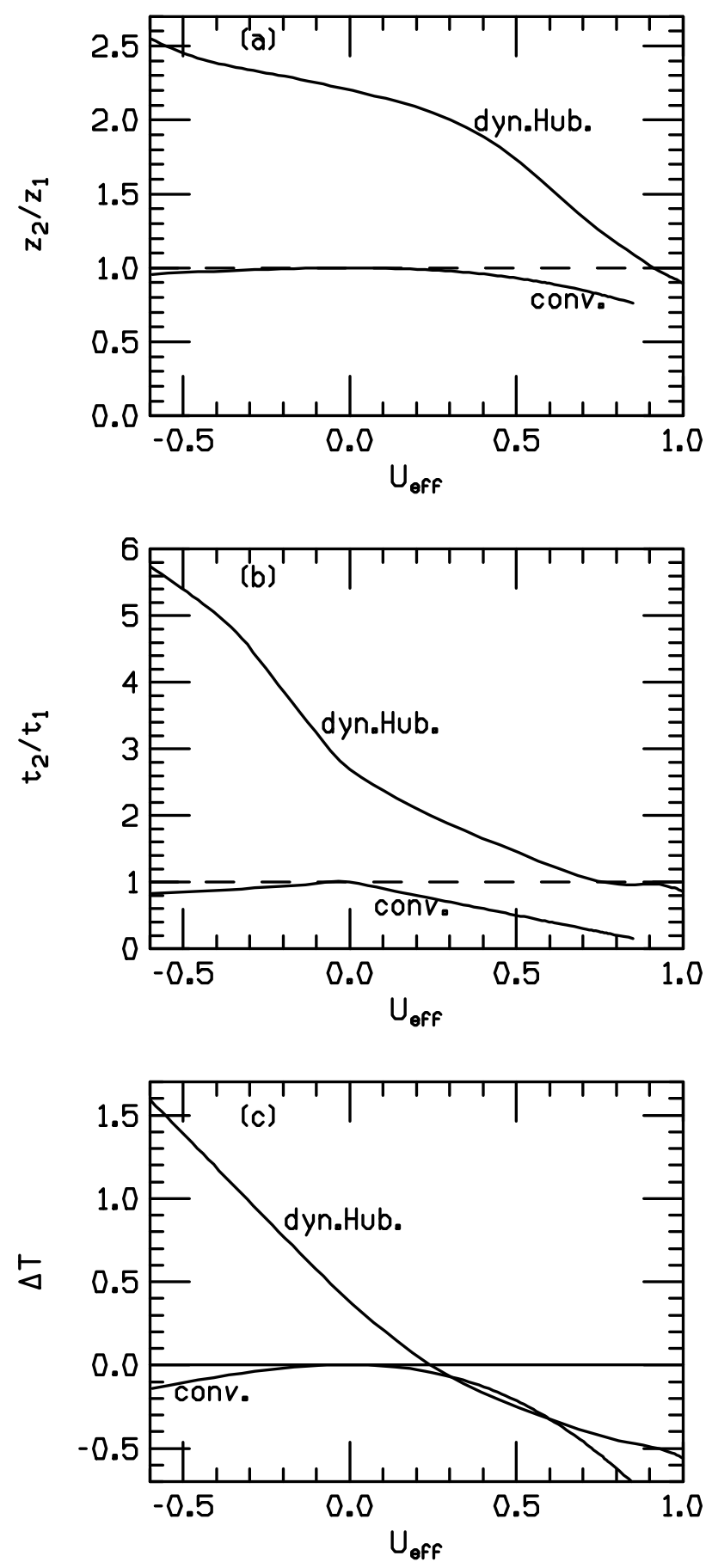

Figure 12 

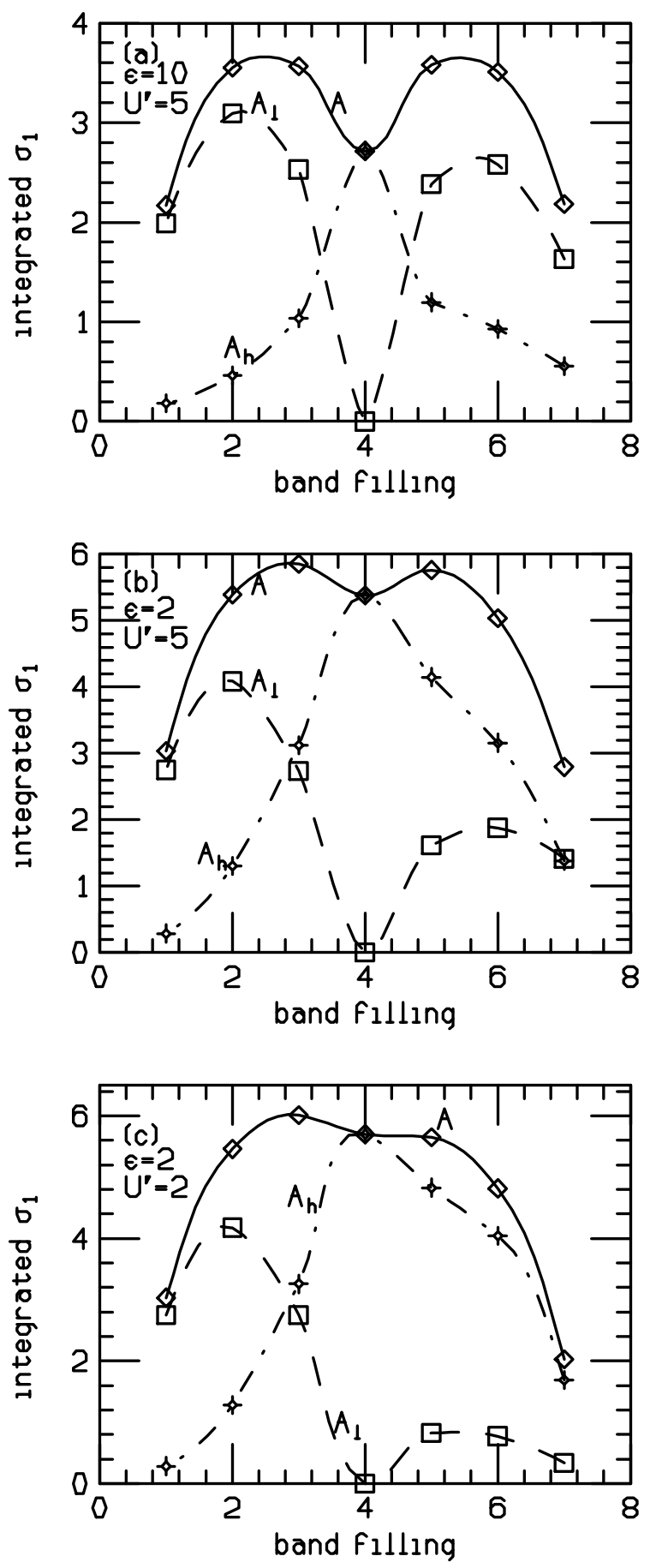

Figure 13 

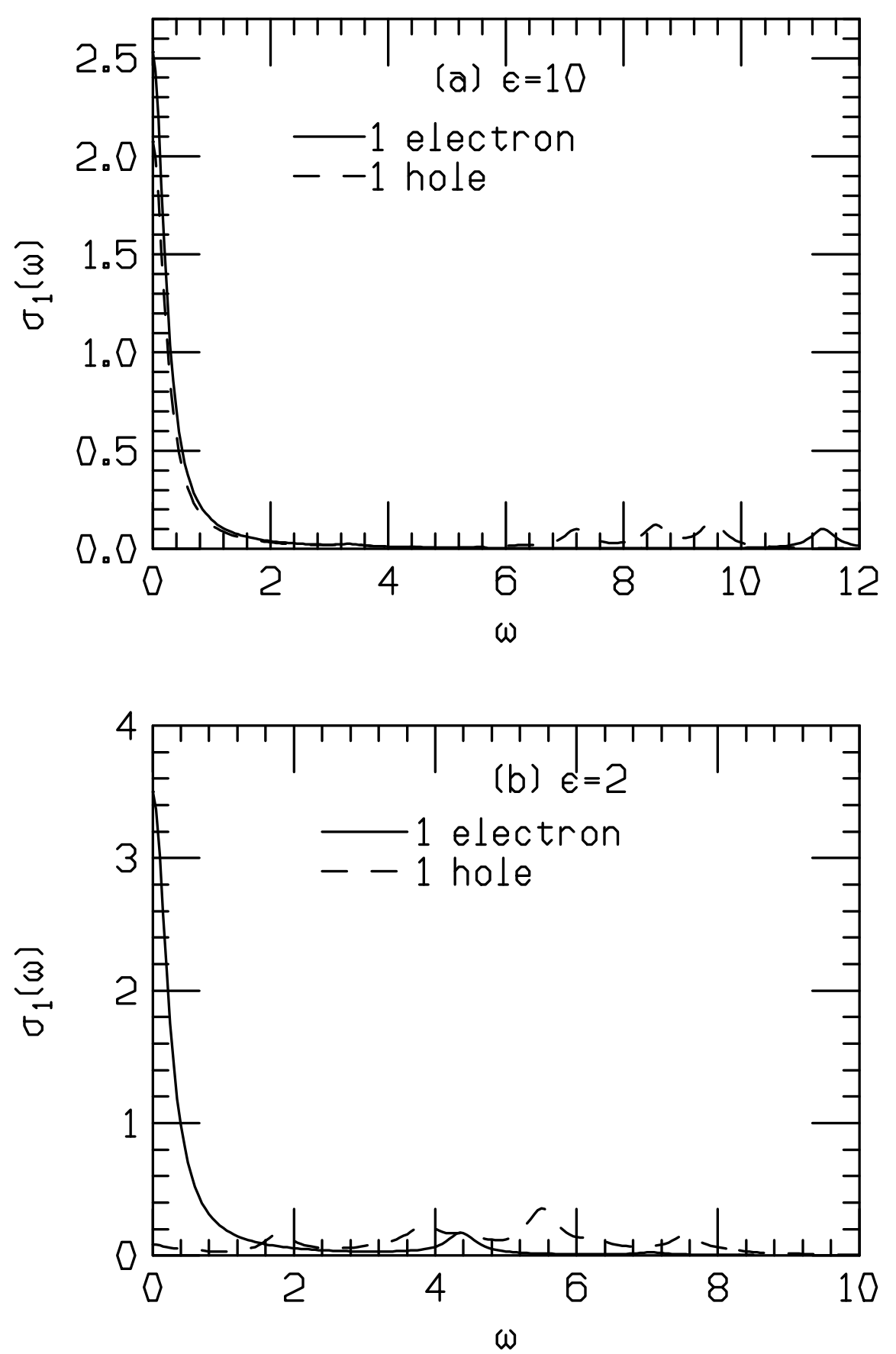

Figure 14 

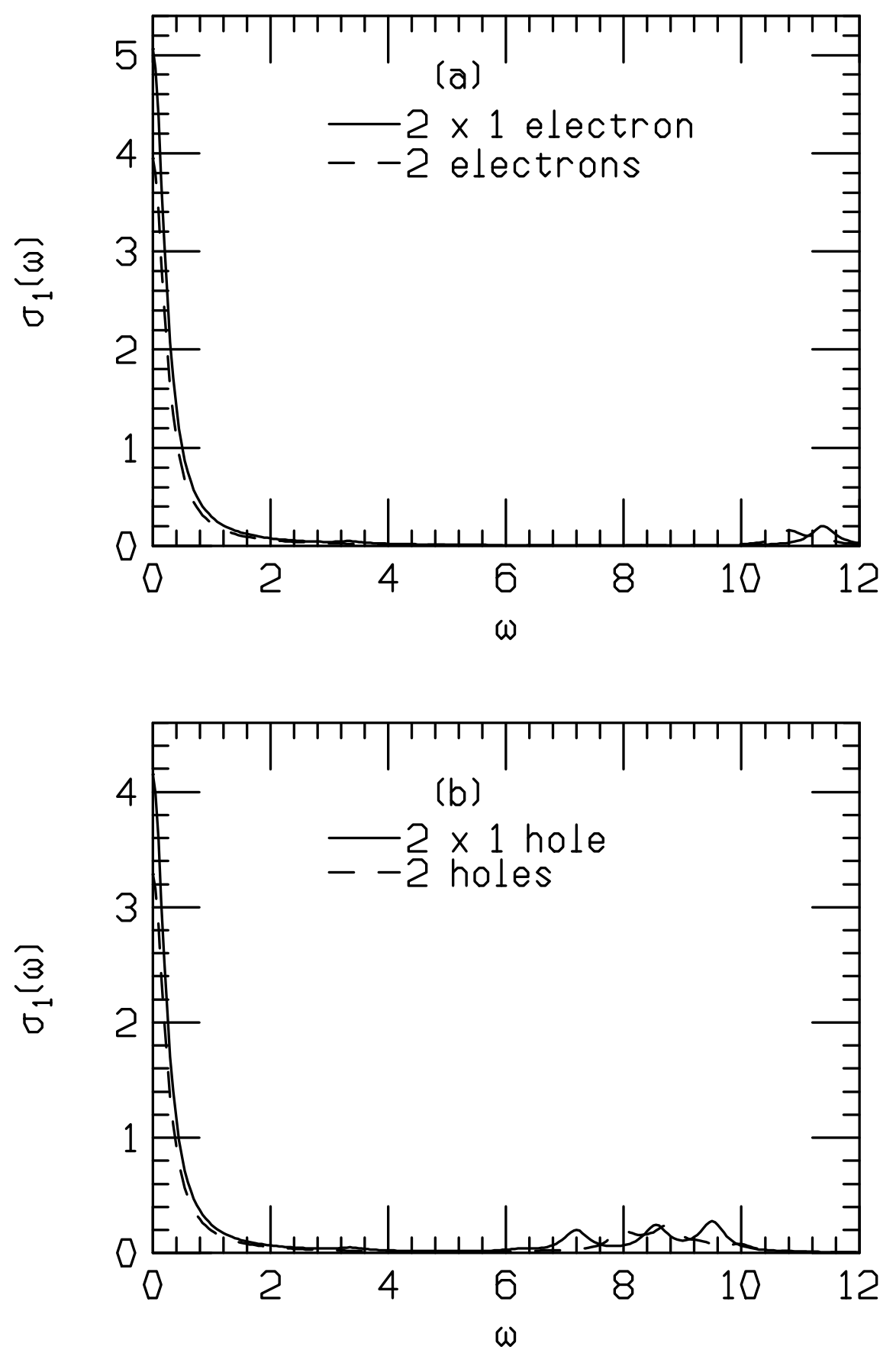

Figure 15 

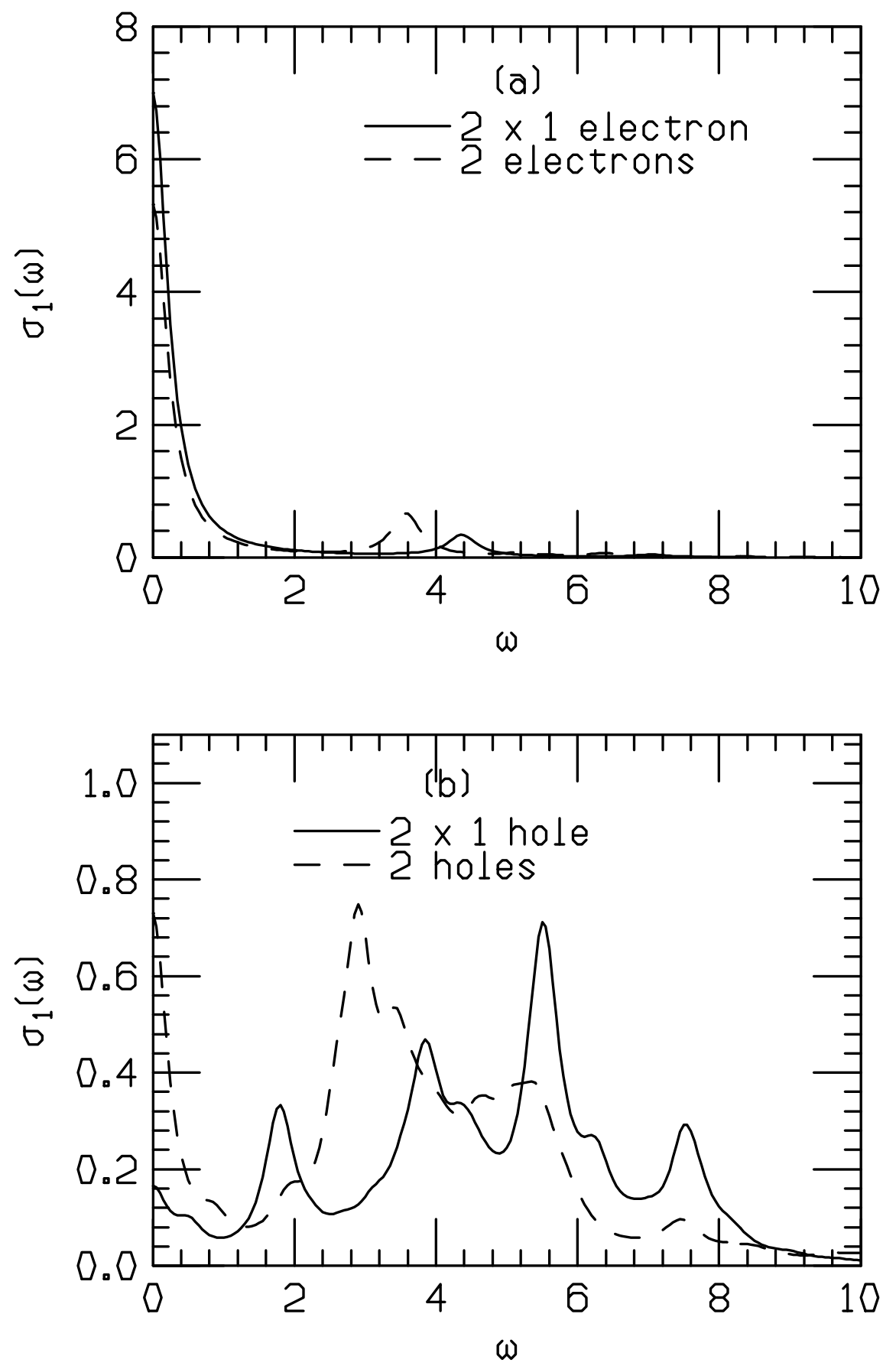

Figure 16 

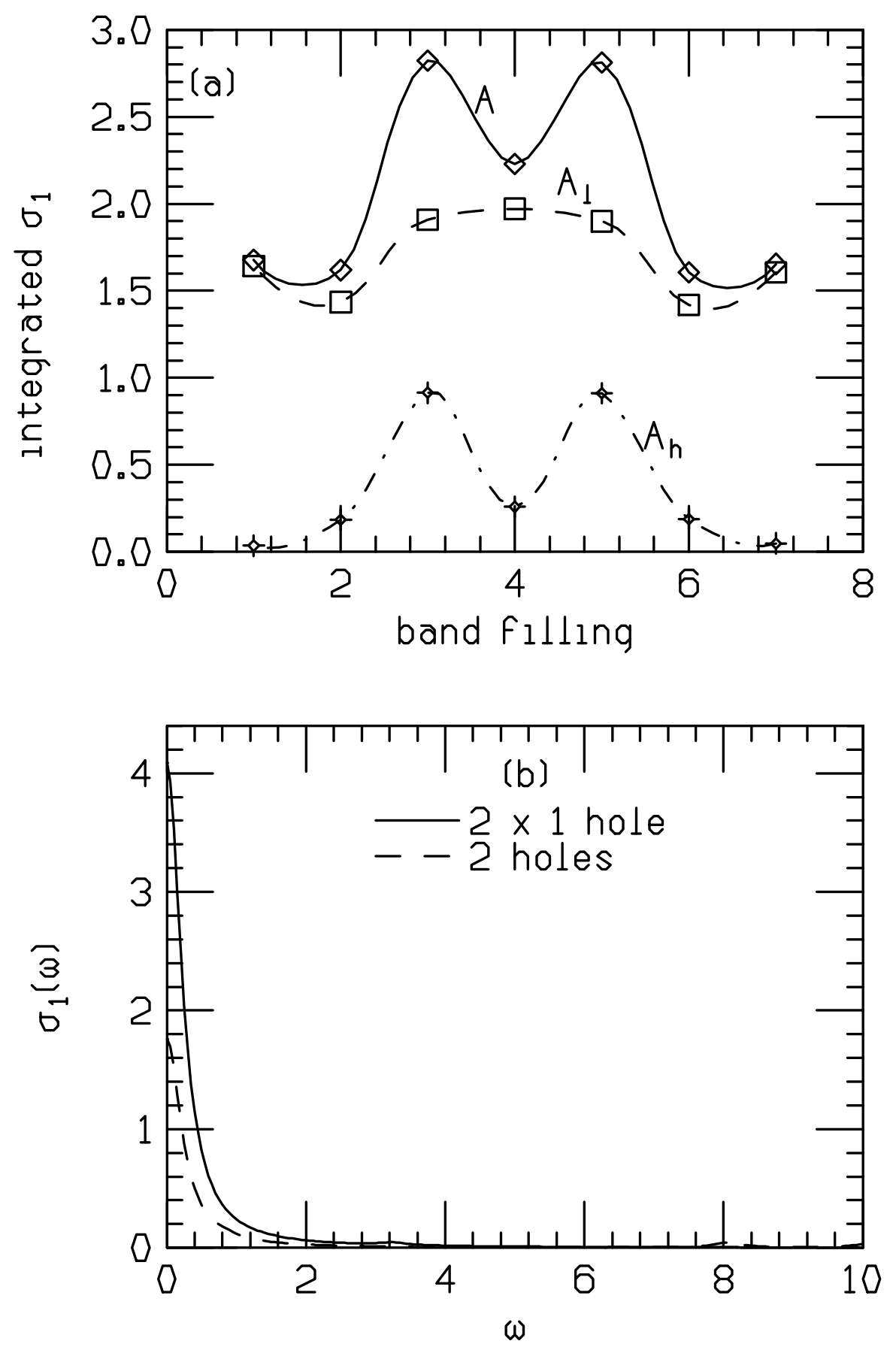

Figure 17 